Illinois State University

ISU ReD: Research and eData

Theses and Dissertations

$4-21-2021$

\title{
Socialization and Civic Engagement in the Virtual Field of Video Games
}

Jackson C. Schwalb

Illinois State University, jschwalb@rocketmail.com

Follow this and additional works at: https://ir.library.illinoisstate.edu/etd

\section{Recommended Citation}

Schwalb, Jackson C., "Socialization and Civic Engagement in the Virtual Field of Video Games" (2021). Theses and Dissertations. 1468.

https://ir.library.illinoisstate.edu/etd/1468

This Thesis is brought to you for free and open access by ISU ReD: Research and eData. It has been accepted for inclusion in Theses and Dissertations by an authorized administrator of ISU ReD: Research and eData. For more information, please contact ISUReD@ilstu.edu. 


\section{SOCIALIZATION AND CIVIC ENGAGEMENT IN THE VIRTUAL FIELD OF VIDEO}

\section{GAMES}

\section{JACKSON SCHWALB}

\section{Pages}

The purpose of this research is to highlight the potential of video games to be a new space for social and civic interaction, facilitated by a variety of structural and community-based features. The theoretical basis for this analysis comes from Bourdieu's theory of practice, explaining the relationship between individual actions and dispositions, the social setting they are in, and the use of his four forms of capital during the interaction. This thesis walks through the production of norms and dispositions of individuals when playing video games, producing their overall habitus in the space. This is followed by analysis of the virtual field of video games, looking at the design and development of these games, the relationship with the video game community at large and the importance of hierarchy and structure in producing a virtual field of interaction between players. The final chapter breaks down the four major forms of capital outlined by Bourdieu (economic, cultural, social and symbolic) with the fourth being adjusted as a new term, gaming capital, as a representation of the combination of the other three forms when engaging in video game play. The results of this research point to the exponential opportunities for video games to facilitate social and civic engagement, as well as producing varying forms of capital that can be used both within the context of the virtual world and the real one. KEYWORDS: civic engagement, cooperation, gaming capital, habitus, immersion, social field 
SOCIALIZATION AND CIVIC ENGAGEMENT IN THE VIRTUAL FIELD OF VIDEO GAMES

JACKSON SCHWALB

A Thesis Submitted in Partial Fulfillment of the Requirements for the Degree of

MASTER OF SCIENCE

Department of Politics and Government

ILLINOIS STATE UNIVERSITY 
(C) 2021 Jackson Schwalb 
SOCIALIZATION AND CIVIC ENGAGEMENT IN THE VIRTUAL FIELD OF VIDEO

GAMES

JACKSON SCHWALB

COMMITTEE MEMBERS:

Julie Webber, Chair

Meghan Leonard

Kam Shapiro 


\section{CONTENTS}

Page

CHAPTER I: SOCIAL NORMS AND HABITUS IN A VIRTUAL WORLD 1

Introduction $\quad 1$

Literature Review $\quad 2$

Bourdieu and Constructivism: A Breakdown of the Norm Production/Diffusion

$\begin{array}{ll}\text { Process } & 2\end{array}$

Toxicity of Video Games $\quad 8$

Cooperation and Problem-Solving in Video Games $\quad 10$

Analyzing the Habitus of Video Games 13

Eliminating the Toxic Video Game Culture 13

Positive Personal Development through Game Play 18

Video Games and their Influence on our Everyday Lives 23

$\begin{array}{ll}\text { Conclusion } & 28\end{array}$

CHAPTER II: STRUCTURE OF THE VIRTUAL FIELD 30

$\begin{array}{ll}\text { Introduction } & 30\end{array}$

Literature Review 33

Importance of the Social Field's Structure 33

Flow Theory, Immersion and Interactivity 36

Virtual Social Field of Video Games $\quad 42$

Positive Impacts of the Competitive Gaming Environment 42

Social and Civic Value of Playing MMORPGs 49

$\begin{array}{ll}\text { Conclusion } & 57\end{array}$ 
Introduction

Literature Review $\quad 61$

$\begin{array}{ll}\text { Social Hierarchy and Class Position } & 61\end{array}$

Hierarchy in Video Games $\quad 66$

$\begin{array}{ll}\text { Social and Civic Hierarchy in the Virtual Field } & 72\end{array}$

Building Social Structures in MMORPGs 72

Competitive Ranking Systems’ Hierarchal Structure 80

$\begin{array}{lr}\text { Conclusion } & 84\end{array}$

CHAPTER IV: PRODUCTION AND UTILIZATION OF CAPITAL IN ONLINE GAMING 87

$\begin{array}{ll}\text { Introduction } & 87\end{array}$

Literature Review $\quad 89$

Bourdieu’s Forms of Capital $\quad 89$

Forms of Capital in Video Games $\quad 94$

Varying Forms of Capital in Video Games $\quad 99$

Production and Conversion of Economic Capital in Video Games 99

$\begin{array}{ll}\text { Cultural Capital in Virtual Worlds } & 103\end{array}$

Socialization Producing Capital Through Gameplay 110

$\begin{array}{ll}\text { Gaming Capital } & 113\end{array}$

$\begin{array}{ll}\text { Conclusion } & 117\end{array}$

$\begin{array}{lr}\text { WORKS CITED } & 120\end{array}$ 


\section{CHAPTER I: SOCIAL NORMS AND HABITUS IN A VIRTUAL WORLD}

\section{Introduction}

When video games are mentioned, what images do they illicit? What norms and behaviors come to mind? Thanks to their unique and complex design, video games manifest many of the positive and negative aspects of classical sports/competition, advanced computer technology, and social media. Some of the leading voices in the field of computer game research like Craig Anderson find that violent video games facilitate negative norms of aggression, antisocial tendencies and an overall lowered attentiveness, (Anderson \& Buckley, p. 375-376). Other authors, like Adam Lobel argue that video games, especially those played cooperatively or competitively, actually help produce positive social skills, cognitive development and even an outlet for aggression and anxiety that alleviates their affects in the real world, (Lobel \& Granic, p. 76-77). Norms and behaviors shaped by video games, whether positive or negative, reflect the overarching dispositions and tastes in players regarding the types of games they play, their individual play style, and how they interact with others both in the game and in other social settings. These dispositions, according to Bourdieu's theory of Practice, constitute an individual's habitus, which is responsible for guiding our decision making and behaviors within social settings.

This chapter will first set out to establish Bourdieu's theory of Practice and how it, and other important political theories like constructivism, help to explain the process of norm production and diffusion within the virtual field of video games. Following this breakdown, this research will look at the prevailing literature surrounding both the negative and positive norms, behaviors, and dispositions developed during video game play. The analysis section of this chapter will then be broken into three parts, the first of which will focus on the prevalence of 
toxic culture and behaviors within video games and how both game designers and the community at-large are actively working to reduce and punish toxic players. The second section will breakdown how game developers, the community and the media are helping to promote a positive culture and habitus within the game's environment. Finally, the concluding section of this analysis will look at how these norms and dispositions can be converted into other social fields and in turn positively impact their social interactions in that setting. Throughout this analysis, the importance of political ideology and the influence that video games can have on it will also be addressed. The goal of this chapter to is not only to highlight the duality of the positive and negative cultures that arise within video game communities, but also to explain and analyze how the habitus that is produced can positively influence in other social, political, and even economic settings.

\section{Literature Review}

\section{Bourdieu and Constructivism: A Breakdown of the Norm Production/Diffusion Process}

Before discussing the positive and negative cultures associated with video games, we must first establish our theoretical support for this chapter. The overarching approach used in this thesis will come from Bourdieu and his theory of practice, which asserts that knowledge, and the actions that spawn from that knowledge, are constructed through social interaction. This is not a passive intake of the information available, but rather it is an active and flexible process that can produce nearly unlimited opportunities for engagement. Individuals can mimic the actions and knowledge of other actors, but to adequately facilitate the production of the practice Bourdieu is talking about, an individual must be interactive and responsive to the environment. Bourdieu's theory can be illustrated as (Habitus $\mathbf{x}$ Capital) + Field = Practice). This formula displays the importance of habitus and its interdependent relationship with the varying forms of capital that 
we both inherit from others and develop through social interaction. This relationship is also largely dependent on the setting and perception of the social interaction, in other words the field in which the actors are operating. The combination of these produces our everyday practices and behaviors that we feel are normal or at least in-line with social and political expectations. The importance and influence of both the field and forms of capital will be addressed in the later chapters, but for now let us focus on the concept of habitus and how it relates to the concept of constructivism.

Habitus, according to Bourdieu, operates as "not only a structuring structure, which organizes practices and the perception of practices, but also a structured structure; the principal division into logical classes which organizes the perception of the social world, which is itself the product of internalization of the division into social classes," (Bourdieu, Logic of Practice, p. 171). What Bourdieu is describing here is an overarching disposition towards understanding our own behaviors and the behavior of others as well as how those behaviors fit into the preestablished norms and rules in a specific social environment. It is all about perspective of others and their tastes in relation to your own and how that impacts the social engagement between you. Habitus in the context of video games can have an impact on a number of different aspects of play like influencing the types of games we play, how and why we play those games, and how we choose to interact with others in the virtual environment, both real people and NPCs (non-playable characters, generated by the game). These interactions facilitate a collective set of rules and norms for the video game community that individual players are inclined to follow. One of the most important aspects for understanding and analyzing the development of an individual or collective habitus is that it is beyond the control of the will of the individual or the community at large. Rather it is a subconscious orientation of practices based on the norms and 
values established, (Mahar et. al., p. 10-12). This does not mean, however, that a person's will or the structure of the setting cannot influence the development of habitus. In fact, it highlights the importance of the preestablished norms and values that dictate the social interaction. The movement of these norms between social spaces is essential for this argument and the foundational aspects of constructivism help us to better understand how this diffusion and reconfiguration happens.

Constructivism, outlined by Ted Hopf, is concerned with "analyzing the interdependent relationship of structure and agency. Attempting to determine how much of the structure is able to constrain the actor as well as how much the actor is able to deviate from those constraints," (Hopf, p. 172-173). Hopf also points out that to better understand the motivations of individual agents, we have to incorporate their discursive powers into the analysis. An individual's ability to be successful in social environments is largely dependent on their ability to manipulate the situation to their benefit through indirect means. Using knowledge, culture and ideology as forms of power compared to more brute force forms. To be more specific, this analysis will utilize a form of constructivism known as social constructivism, which posits that human agents actively construct and reproduce their social reality through their everyday actions and choices. Risse, pointing out the argument from March and Olsen, states that social constructivism uses a "Logic of Appropriateness" when trying to understand human behavior, which states that human actors are imagined to follow rules that associated particular identities to particular situations, approaching individual opportunities for action by assessing similarities between current identities and choice dilemmas and more general concepts of self and situations (Risse, p. 5). What is so important about this form of logic is it helps explain the wide variety of possible social interactions with which an individual can engage a situation. Each social situation can be 
different in terms of setting, context, individuals involved, and even the capital produced or converted during the interaction. This means we must be able to recognize the influence of the intangible aspects of socialization like a person's disposition, tastes, style choices, etc., all culminating into their individual, and the settings' collective habitus. We must ask ourselves, however, what about this explanation makes social constructivism relevant to video game play and to a larger extent, video games' impact on our social, cultural and political decisions?

According to Vittorio Marone, understanding and providing a conceptual framework for digital play through the lens of learning requires utilizing the dimensions of play, design, and participation in the analysis. In his breakdown of the concept of play, Marone posits that, due to the unbounded and expansive nature of the possibilities available in video game play, digital games embody a dual characteristic of a challenging and tutorial environment in which players/learners are presented with problems, tasks, and missions that are progressively adjusted to match their current level of competence, (Marone, p. 3-5). These games operate as dynamic systems, through which the players interacts with a wide variety of elements that are in turn interacting with one another in response to the overarching rules and norms set by the game's designers, the commands being controlled by the AI, and the basic inputs of the player. The makeup of these systems varies wildly however, with largescale hyper-systems at the macro level and subsystem at the more individual level. Hyper-systems refer to the culmination of designers, critics, developers, and the game's online community, which facilitates a general identity for the stakeholders involved. Sub-systems are community groups spawned from the hyper-system established with the development of the game. World of Warcraft's (hereafter WoW) guild system is a great example of a sub-system operating as an advancement of positive socialization, individual and cooperative advancement within the game and even an avenue for 
learning important skills and techniques that can be applied outside of the game as well, (Marone, p. 6-7). WoW guilds, and other manifestations of subsystems in video games, provide players with a social setting that not only positively influences their social interactions in game, they also help minimize the negative and toxic culture generally associated with video game play.

The design features of video games, according to Marone, are also essential in establishing the positive social interaction within and outside of the game. Game design, especially through the "modding" and editing process, allows players to manipulate their ingame environment to facilitate the development of the proper norms and values, (Marone, p. 7-9) By providing players and students (through the application of these strategies to schooling) with this type of structural freedom, their ability to understand how these settings are constructed, both through social and technical terms, as well as making adjustments to the current environment to better fit their needs. The final aspect of video games that is essential for Marone is presence of participation within these games. Participatory or cooperative play is integral in the learning process, helping students conceptualize the importance of working together to achieve goals as well as providing them with a peer support system that they may need later on. These positive aspects, on top of the positive socialization that the students are receiving, produce an environment that directly increases a student's ability to in-take, process and utilize the information provided to them. During a participatory process, especially in a video game environment, a setting known as an Affinity Space is produced, which creates a participatory culture that contains, "relatively low barriers to artistic expression and civic engagement, strong support for creating and sharing creations, and some type of informal mentorship whereby experienced participants pass along knowledge to novices," (Marone, p. 9-10). This description 
highlights the positive impact social communities like guilds in WoW can have on new players. This affinity space in turn can facilitate the conversion of cultural knowledge and competence, as well as collaboration/cooperation strategies, into other social spaces that this information can be useful in.

As I mentioned above, arguably the most crucial aspect of video game development for this analysis is their ability to produce, reproduce, and facilitate the conversion of norms and values in other social fields. The biggest knock on video game development is its negative effects on our socialization and aggression levels, but as Adam Lobel and other supporting authors highlight, video games actually positively impact our social interaction skills, cognition levels, and mediates our aggression and antisocial tendencies. The final section of the analysis portion of this chapter will look at how these skills developed through play can be and are converted into other social settings like school, work and civic engagement making us better equip to perform in these settings. Before moving into the analysis, however, both the negative and positive cultures and dispositions correlated with video game play must be established and better understood. In the next section, I will breakdown the prevailing literature regarding the negative, toxic, and aggressive culture most commonly associated with video games as well as explaining where these studies were limited in their scope and replicability. Following this examination, I will highlight the more recent research into video game play, specifically the explosion of MMOs (mass multiplayer online games) and how the impact of cooperative and competitive play has shifted the narrative around video games and what that means for their development of positive norms and values. 


\section{Toxicity of Video Games}

Before breaking down what the toxic culture of video games looks like specifically, we must first analyze the prevailing literature regarding this subject. One of the premier voices in field of video game research, Craig Anderson, has spent the last 30 or so years trying to understand how and why toxic and aggressive behavior manifests in video game play and what some of the factors are that contribute to this culture. In one of his most important studies, he and his co-author Brad Bushman find significant evidence of media violence facilitating the production of aggression, antisocial behaviors and addictive tendencies, (Anderson \& Bushman, p. 354-355). Through this analysis, violent video game play was found to be associated with a significant increase in aggressive thoughts, regardless of age or gender, and a negative correlation with prosocial skills in the real world, (Anderson \& Bushman, p. 358).

This data is an important starting point for understanding when the negative connotation surrounding video game play first arose. It highlights the general public perspective of violent video games as well as some grounded, negative aspects associated with this kind of play. This study, however, suffers from a number of validity issues, with the most prominent being the game utilized in this study. Much of Anderson's research focused on outdated examples of video games, most of which were arcade based or even solo-player games. The issue with this, which I will go into more depth on in the next section, is the complete disregard for the influence of cooperative play games. This study does come from 2001, meaning that the games on the market at the time did not clearly represent the positive social and emotional experiences that the teambased games in the current times have to offer. Anderson has even in recent years updated his research to include these positive social aspects but has not walked back his argument regarding aggression. In his 2006 article co-authored with Katherine Buckley, the authors provide evidence 
of the prosocial development, highlighting the ability of cooperative games to produce problemsolving, teambuilding and empathy skills that can then, in turn, be converted into other social fields like school or work. The authors do stick with the argument of increased aggression however, pointing out that certain input variables in these settings can directly influence our mood and emotions. Video games contain a number of triggering variables like high levels of arousal and exposure to frustrating or confusing scenarios in-game, (Anderson \& Buckley, p. 371-373). These inputs are an important aspect of video game play but can cause players to develop an overarching disposition towards toxic behavior within the game which can, in turn, be carried into other social environments. This creates both an individual and collective habitus for these gaming communities that makes the player inclined to engage in this toxicity.

So, what does this toxic culture look like? According to Adinolf and Türkay's article, toxic behavior can come in the form of griefing (intention disruption of another player or destruction of their property), raging (explosive verbalization of frustration), cheating, cyberbullying or trolling, racism, and misogyny. These, according to the authors, are examples of deviant play form which is defined as, "those which fall outside of the bounds of play contracts, both designed by the game company and the social norms within the game's community and highlights the constant negotiations about the boundaries and meaning of play," (Adinolf \& Türkay, p. 366). These aggressive, hateful and disruptive behaviors are most commonly directed individuals regarding the race, religion, sexual orientation and even gender. The ability to remain relatively anonymous in these environments has given toxic individuals in society a platform to exercise their frustrations on other players with little to no recourse. In the first section of the analysis portion of this chapter will look at how both the game's developers and the community at large have worked to curb toxic and hateful behavior. Before do that however, we need to 
discuss the parallel habitus forming around video game play, which that of positive social and cognitive development that promotes social engagement, cooperation, problem-solving and critical thinking.

\section{Cooperation and Problem-Solving in Video Games}

Now that we understand the stereotypical negative connotations associated with video games, the focus can shift to more contemporary concerns. In recent years the development of video games, especially MMOs (mass-multiplayer online), has exploded making it one of the most profitable and influential sources of media on the planet. The scope of the video game community and the culture that comes with it is nearly unlimited, from thousands of different games and genres, streaming and video platforms like Twitch and YouTube and the intricate development of the eSports leagues for various game franchises. All of these are examples of the reach that video games can and do have around the world. That being said, the negative assumptions the public has held regarding video games (i.e. addictive, aggression-producing, antisocial tendencies) are no doubt stoked by sensational journalism and have hindered this development and blinded the public in many ways the to the important social and cognitive skills that video games can provide to their players. These skills, in addition to the positive norms and values like cooperation, empathy, time management, etc., facilitate the development of a more successful and efficient member of society through the conversion of these skills, norms and values into other social settings like school and work. In their article, when referencing the cognitive benefits of video games, Adam Lobel and Isabela Granic state that, " The most convincing evidence comes from the numerous training studies that recruit naive gamers (those who have hardly or never played shooter video games) and randomly assign them to play either a shooter video game or another type of video game for the same period of time. Compared to 
control participants, those in the shooter video game condition show faster and more accurate attention allocation, higher spatial resolution in visual processing, and enhanced mental rotation abilities," (Lobel \& Granic, p. 68). They go on to point out research from 2013 that displays evidence of the spatial skills developed while playing video games are comparable to those developed in high school and college level courses, (Lobel \& Granic, p. 70). This is clear evidence of the cognitive advantage that can be gained by playing video games, both as a leisure time activity and as a tool within school or work (something I will discuss at length in the analysis section). Improvements in cognitive skills are not the only positive to focus on however, as the social interactions aspect of video game play may be the most influential for this discussion.

In their analysis, Douglas Gentile and his co-authors find significant evidence of positive prosocial development in individuals who participated in cooperative video game play as opposed to either a neutral or violent game. Their data presented them with evidence that participants that played prosocial games were found to be statistically more likely to engage in helping behaviors outside of the context of the video game than the other two groups. Additionally, those players that engaged in the violent games were far more likely to perform hurting behaviors as opposed to helping. Finally, they found a direct correlation between the incorporation of prosocial aspects into a game, regardless of the level of violence, and the individual's willingness to engage in helping behaviors increases dramatically, (Gentile et. al., par. 15-17, 21-22 \& 29-35). Gentile's research leads him to establish five dimensions of video game play that have a direct impact on the player, which are the amount of the game played, content of the game, context/setting of the game, structure/design of the game, and finally the physical mechanics of the game, (Greitemeyer et. al., p. 1466). The variation and influence of 
these factors is largely what determines the impact of the game itself, not simply the presence of violence or addictive content.

So, what does this data mean for our understanding of the culture of video games and the general dispositions of players? It tells us that the violence within the game itself is not the defining feature. The impact that video games have is far more complex than that. They provide players with a social environment to have both positive and negative social engagements while simultaneously training skills of cognition, problem solving, and critical thinking, among others. In the remaining portion of this chapter, I will breakdown first how game developers and their communities are working constantly to remove the toxic culture and stigma associated with video games and what that means for the design of the games themselves. Second, we look at the concurrent role that both the developers and community play, which is promoting the positive social norms and values that they want to establish as the standard within the video game world. This requires systematic changes to the way games are built as well as shifting the public perception through media campaigns that highlight the prosocial aspects of multiplayer games. Finally, the conclusion of this chapter will look at the ability of video games to reproduce existing positive norms from other social settings, produce their own new norms, and facilitate the conversion of them back into the other important social environments in our lives like school, work and even civic engagement. By the conclusion of this chapter, a more defined representation of the positive impact that video games can have on other social, cultural, and political settings will be established. 


\section{Analyzing the Habitus of Video Games}

\section{Eliminating the Toxic Video Game Culture}

As mentioned above, toxic behavior in video games can manifest in a variety of forms ranging from griefing to hate speech. These behaviors all contribute to an overall disposition of the players within the game's community to engage in and reproduce toxic interactions. Women and racial minority groups have had the vast majority of toxic chat or in-game behavior directed towards them. According to Dietz's article, video games, not unlike other forms of mass media, reinforce gender stereotypes that place specific masculine and feminine expectations on individuals in social settings, (Dietz, p. 428-429). These direct attacks on female players and feminine culture in general makes the norms and values established through this process inherently political. The toxic and hateful rhetoric spread through the medium of video game can have both a direct and indirect influence on the player's political ideologies as well as their level of empathy towards other, unrelated groups.

Through his analysis, Dietz's finds significant evidence of this disproportionate representation of women in video games, stating that only $15 \%$ of the 33 games sampled had female heroes, many of which were dressed in revealing and sexualized outfits. Additionally, the author finds non-inclusion (30\% of games) to be most common decision regarding female characters with the "Damsel in Distress" (21\% of games) being the second most common, (Dietz, p. 433-437). The consistency with which the stereotype of the weak, useless woman is utilized within video games has periphery effects on the individuals who are exposed to this content. It encourages male players to view and treat women as such and leads to toxic behavior directed at female players. Due to this deep seeded culture of toxic masculinity, women have been forced to be more alert and perceptive of hateful and sexist interactions when playing 
games. In her article, Amanda Cote points out that of female gamers surveyed, over $37 \%$ have experienced some form of discrimination. To combat this, Cote has suggested a number of personal strategies to help avoid these types of interactions, some of which included muting or disabling the in-game chat, not revealing your gender or race, and even simply getting better at the game to "shut up the competition," (Cote, par. 6-9).

The suggestions made by Cote, while providing some short-term solutions for individual players, do not address the systematic problem of a toxic and pervasive culture surrounding video games. This cannot be solved at the micro level, rather the games' developers and moderators have to actively work to curb all forms of toxicity on their platforms. Riot games, developers of the extremely popular franchise League of Legends, have had to work extensively to try to eliminate the hate speech, griefing and cheating taking place in their game. Riot decided to engage in three experiments to determine how they can best combat their issue of toxic behaviors, the first of which simply provided players with the option to mute or disable their ingame chat. Even though it was only provided as optional, the change lead to a $32.7 \%$ reduction in negative chats over a one-week span and $34.5 \%$ increase in positive chats over the same span of time with no reduction in chat usage overall, (Soderberg-Rivkin, par. 6-8). This experiment highlights the possible effectiveness of the suggestions made by Amanda Cote in her article. Through your own self-mediation of the social interactions within the game's environment, you can actively reduce the exposure and influence of negative chats and behaviors.

Riot's second experiment focused on providing players with a systematic process for reporting and punishing toxic and abusive behaviors. They introduced the "Tribunal System" which essentially gave players a public forum to report instances of toxic interactions to both shame the player in question as well as seek punishments like bans or suspensions from the 
game. It places a "reform card" on the player's profile to highlight the behaviors that got them in trouble as well as an appeal system to possibly be reinstated. This system has been proven to be extremely effective for Riot Games, with high levels of usage for the Tribunal System being reported weekly, (Soderberg-Rivkin, p. 9-12). It also highlights how effective a public forum system can be in the context of video games as it provides players with an opportunity to engage civically and seek justice through League of Legend's laws and regulations. The final experiment is the focused on providing primers to players to encourage positive behaviors and steer them away from negative ones. To test this, the developers randomly selected the pre-game messages for players (either positive, negative or neutral) and their overall shift in type of toxic chat was recorded. Those that were exposed to positive messages recorded $6.3 \%$ decrease in verbal abuse, $5.9 \%$ decrease in offensive language and $4.1 \%$ decrease in in-game reports, (Soderberg-Rivkin, par. 15-17). All three of these experiments display how effective a video game developer can be at actively reducing the amount of toxic chat and behaviors in their own game. Utilizing their player base to test the efficacy of their new approaches proved to be extremely useful in determining what constitutes toxic and abusive interactions as well as what tools can be effective at stopping it.

Another great example of the effectiveness of a game's developer at reducing hateful and toxic behavior within a game comes from Minecraft. Minecraft is a blocky, open-world building game that provides the player with nearly unlimited activities and objectives. Video games that contain similar structure and mechanical features to Minecraft are known as "sandbox" games, as they give a player a vast degree of creativity in terms of objectives, resources and architecture, much like a real-life sandbox. Minecraft was one of the earliest examples of a sandbox game and set the standard of an unrestrictive format for providing objectives, storylines, etc. to the player. 
Minecraft places the individual in a random spawn point on an infinitely generating map in which players must collect resources, build tools and a home, begin farming/mining and fighting monsters, all in an effort to survive. Players can do any and all of the tasks, jobs, etc. provided to the player or can simply stick to one like fishing or mining. This provides levels of selfdetermination and individual liberty unheard of open world games. This has become one of the major selling points for Minecraft over the past ten years, but it has also spawned the widespread issue of griefing in-game. Due to the lack of restrictions on the actions of players in most Minecraft servers, players tend to engage in the destruction of others' property or trolling other players by stealing and hiding items. This has caused a huge divide in the community according to Rolfes and Passig, who report that roughly $50 \%$ of players think griefing should be removed from the equation and 50\% who believe it is a normal aspect of playing Minecraft, (Rolfes \& Passig, p. 3). Minecraft's developers have always wished to maintain a hand-off approach to moderating the in-game environment, only looking to step in if it is a systematic issue rather than a player-to-player one. The rules and parameters established by developers constitute the "digital institutions" of the game but cover very little in regard to interpersonal interactions. With the lack of proper limitations on individual player behavior, the developers were forced to offer tools to server owners and moderators to help mediate these situations.

These tools, according to Rolfes and Passig, are a "large collection of plugins that automate the deployment and maintenance of rules for peer monitoring, resource monitoring, rule enforcement, trade, vandalism, decision-making, information transmission, and communication, as well as plugins that define complete market and property rights institutions: systems of property rights, shops, social hierarchies, and group allegiances," (Rolfes \& Passig, p. 6-7). These plugins have given power to those in-charge of the servers, reducing the 
responsibility of the developers to oversee the micro-level interactions. However, this process has had unintended consequences in the form of proto-governance systems arising within these servers. According to Rolfes and Passig, the most likely form of government to manifest in these servers is either a benevolent dictatorship or a constitutional democracy. This is due to the lack of democratic institutions like electing leaders or participatory forms of civic engagement built into the design and rules of these servers. In the benevolent dictatorships, server owners, although they rule with near unchecked authority, promise to exercise their unlimited power for the good of the community and refrain from abuses of power. However, server rules are often limited and there is very seldomly proper plugins in place to mediate the power of those in charge. In the case of the constitutional monarchy, some rulesets do place limitations on the power of both moderators and the owner of the server. These servers still lack constitutional features necessary in democratic institutions but do have rules in place that punish leaders for toxic or self-interested behaviors. Constitutional monarchy servers also establish a penalty system for players that do violate the rules, all of which would be proportional to the crime committed, (Rolfes \& Passig, p. 8-11).

Now while this article highlights the extensive negative possibilities that can arise from these Minecraft servers and their semi-unregulated nature, there are some positives that can be pulled from it. Firstly, the advent of plugins provides an avenue for possible future adjustments to the game's design that could lead to further reduction of toxic behaviors. The plugins, and the willingness of Minecraft's developers to step in and take a more active hand in moderating servers, can also instill positive habitus in its player base that produces trust in those in-charge of the social setting as well as the structure of it. On the other hand, exposure to centralized and ineffective modes of governance in this virtual context helps those playing to understand why 
they how they are unrepresentative and cannot meet their needs in a real-life context. These centralized forms of governance arising within video games are not a coincidence, as we see authoritarian regimes arising around the world. The individuals in charge of these servers saw a chance to attain power over a social environment in the same way autocratic leaders seized their opportunity. The point however is the masses within these social contexts, whether in be in the real world or a virtual one, are recognizing the inability of the leadership in these structures of governance to meet their wants and needs. Players in these Minecraft servers have spoken out and asked for the changes we are already seeing the developers make, showing how these players are finding their voice within a community and becoming civically engaged. In the following section, we will look at the dual role that both video game developers and the video game community take on, which is the promotion and facilitation of norms and values associated with cooperation, problem-solving, and empathy towards others. All of these work in tandem to produce a more positive habitus for the community.

\section{Positive Personal Development through Game Play}

Now that the toxic culture surrounding video games has been addressed along with the active steps being taken to minimize it, we need to shift focus towards the positive norms and values that are produced and reproduced through video game play. Returning to the discussion of Lobel and Granic's piece, the major areas that are positively influenced by video game play are an individual's overall cognition levels and spatial skills, development of prosocial skills and promotion of social settings, and an outlet for aggression and aggressive thoughts, (Lobel \& Granic, p. 71-74). In line with Lobel and Granic's thinking, Halbrook and his co-authors engage in a similar study to determine the possible outcomes of video game play. What they found was directly supportive of Lobel and Granic's research, while expanding past it to include 
"Exergames" (i.e. Wii Sports, Xbox Kinect) as another positive avenue of video games. The authors concluded, in terms of the social benefit, that players who engaged in MMORPGs (massive multiplayer online role-playing games) with socialization as the primary motivator, reported significantly lower levels of psychological symptoms like depression or anxiety than those that played too long or alone. The social engagement aspect also helped to provide "remote socialization" for individuals who lack the skills or the proper social setting necessary to create positive socialization and decrease depression and anxiety. In terms of the violent content incorporated into video games, the authors found a significant decrease in a player's willingness to engage in violent or aggressive behavior when exposed to violent games when those game include cooperation and socialization variables. These variables have a positive impact on a player's socialization skills and minimizes the overall negative impact of the violent content, (Halbrook et. al., p. 1098-1100).

Both this article and the one from Lobel and Granic provide us with some clear insight as to the active part that video games can and do play in the development of positive norms and values. The genre and design of a game however can influence how these factors can be produced. For example, the impact that the eSports community and the games they play is heavily based on the general motivators for play, which include achievement, socialization and immersion. On top of these, eSports titles offer additional motivators like self-improvement, socialization and competition. According to Carbonie and her co-authors, 79\% of players surveyed reported experiencing all of the values and benefits mentioned. Of the benefits, the three highest recorded were commitment to a task, cooperation, and initiative. In terms of the values provided, the top three were hedonism, power, and purpose in life, (Carbonie et. al., p. 67). These values benefits can be essential to our everyday lives outside of the virtual world but as 
this article displayed, they have a clear and influential impact on the virtual social field of video games. To better understand what these values look like in practice, let's look at the development of the Role Queue System in Overwatch's Ranked Play System.

As a bit of an overview, Overwatch is a competitive 6v6 FPS (first person shooter) video game that requires extensive team chemistry coordination and cooperation to be effective and win games. The Overwatch community has exploded in the past few years, developing an extremely successful eSports league and possible sequel games to be released. Because of the increase in popularity of the eSports side of Overwatch, the game's developers have incorporated a ranked play system through which players, either solo or queued up with friends, play 10 placement matches and are then sorted into a tier system based on a player generated skill level. They then engage in ranked matches against other in the same tier as them hoping to move up into higher divisions. This process utilizes the ELO ranking system, which was originally developed for chess tournaments in the 1950s but has been proven to be an effective system in video games as well. The Elo Ranking System can be defined as a competitive ranking system using a scale of 0 to 3000 that fluctuates over times based on a combined scored averaged out between a player's wins, losses, and draws, (Ourania et. al., p. 3-4). This ranking system will be an important aspect of this thesis, but the discussion is better saved for chapter two. For now, just know that the ELO system is an integral aspect of the competitive side of Overwatch.

There are three standard character classifications to pick from: healer, support and tank. Each provides their own specific skills and attributes, which become further differentiated when we look at individual characters. Primarily, healers are responsible for staying alive to provide health to damaged teammates or revive fallen ones. Tanks are large characters with far more health than the other two classes and are many used to take a bulk of the damage coming from 
the enemy team and protect the smaller, less strong characters on your team. Finally, the support character classification contains a variety of specialty character with very unique abilities that allow them to do large amounts of damage to the other team or use fast mobility to avoid other attacks. The importance of these roles has shifted over the course the game's life, changing the primary make-up of the competitive games from playstyle, character choice, etc. which all boils down to the current "meta" of the game. The issue with this system, and what the role queue sought to solve, was that many teams were forgoing the use of support players, opting instead to utilize three healers and three tanks, as many of characters in either class were strong enough to provide the damage per second needed to successful and win games. This 3-3 dynamic became a staple of the Overwatch eSports league and almost completely removed the use of the fun and unique utility characters available in the support class. This shift to the 3-3 dynamic was detrimental for a few reasons, first of which was the game itself became unappealing to watch as a spectator of the eSports league. The constant damage, healing and slow progression of the team made the viewing experience boring and hard to follow. Additionally, this dynamic ruined the important cooperative aspects associated with Overwatch, turning teams away from coordinated team pushes, hindering their ability to make creative and flashy plays to disrupt the other team and really stifled the critical thinking of the players involved.

As a response to this, Overwatch's developer Blizzard implemented a new and innovative system to combat it, the Role Queue System. In the role queue system, players are required when loading into a match to select from one of the three categories (i.e. support, healer, or tank) and that will be their role for the remainder of the game. This helps the team dynamic in competitive play to return to the 2-2-2 dynamic that was the standard prior to the 3-3 set up. Players are also given an individual competitive rank for each role, rather than a collective one for the account, 
further incentivizing players to try new characters and learn how to work as a team, (Lowry, par. 1-5, 8-10). Furthermore, Blizzard installed an endorsement system, through which players can promote their teammates as "shot caller" "good teammate" or "sportsmanship" as a way of having active positive social interactions with your teammates and facilitate prosocial development. Both of these systems being implemented can have a clearly positive impact on the socialization of the players in their community. Overwatch's promotion of positive social norms and values is facilitating the development of a positive habitus associated with playing competitively in games like Overwatch. It encourages players to be part of the community, feel engaged and receive positive outcomes from being a member of it.

Shifting gears slightly, let's return to the impact that free roam games like Minecraft can have on our socialization, as Minecraft's influence is directly pertinent to other social fields like school and engaging in civil society activities. During the process of learning, having information provided to you through a social and emotional context helps make the cognitive more efficient and effective. According to Zolyomi and Schmalz's article, social components to learning are a core feature for their experience which can include behavioral therapy, social goals, and variety of learning supports. Why these matter in reference to Minecraft is because the design and structure of the Minecraft world provides the social components necessary to create this type of positive learning environment. The author's find social interaction to be the number one challenge to young children especially through with neuro-diverse conditions like autism. It is one of the primary causes for children and their parents to seek out therapy and Minecraft is proving to be an effective social outlet for these students while still keeping them within the context of a lesson plan, (Zolyomi \& Schmalz, p. 3394-3396). Minecraft has clear social and educational benefits for students, especially those dealing with socialization issues. Some 
grounded benefits to playing these games for educational purposes, according to Minecraft's website are fivefold: helping with personal development, helping students face and combat social isolation, learning cooperation and conflict resolution skills without real world consequences, teaching respectful and responsible online communication skills, and an overall increase in willingness to participate in community engagement, (Bailey, par. 2-9). These are clear examples of the positive influence that playing video games can have on our own personal development and socialization. The promotion of positive norms and values associated with socialization help to create a positive habitus of cooperation, problem-solving, and empathy towards others within the context of the virtual social field. In the final section of this chapter, we will look at how these positive norms and values can then be converted into other social settings to have a noticeable impact on our socialization and development in both our education and in our civic engagement.

\section{Video Games and their Influence on Our Everyday Lives}

Up to this point, this chapter has completed all but one of its objectives. We have established a theoretical framework for understanding the process of producing norms and values and creating a general habitus for social interaction thanks to Pierre Bourdieu and a number of social constructivist scholars. Following this we established both the negative and positive culture, norms and behaviors surrounding video games, as well as provided numerous examples of both. The influence of the game's developers, along with the gaming community at large, was incorporated into this analysis to determine how we actively facilitate these social interactions. Now that all of this has been covered, we must address the final goal of this chapter, highlight the ability of video games to reproduce and convert these norms, values and dispositions into other social settings. This conversion is intended to create a positive impact on our social, 
cultural and political behaviors regardless of the environment we are in. This section will be narrowed down to look at the influence that Minecraft has on other social settings, as it can be impactful in numerous areas requires more in-depth discussion. The positive cooperative and problem-solving skills learned in games like Overwatch can be converted into a number of other social spheres as well, but Minecraft's positive influence is an educational setting are more visible and easier to understand. Minecraft can be used as an integral aspect of the learning process by facilitating a more accessible and efficient educational environment, making it a prime area of analysis.

Minecraft's influence on the learning process is directly related to what is known as Social and Emotional Learning or SEL. SEL involves a, "process through which students acquire and effectively apply the knowledge, attitudes and skills necessary to understand and manage emotions, set and achieve positive goals, feel and show empathy for others, establish and maintain positive relationships and make responsible decisions," (Getting Smart Research Center, p. 3). Some key features of SEL include clearly stated goals, integration with academic standards/rules, inclusion of guidelines for both teacher practices and promoting a positive learning environment. These features highlight a mutually supportive relationship between the agents involved and the structure around them, something social constructivism implores as essential for the process of norm production and diffusion.

Video games, especially Minecraft have shown their ability to facilitate the relationship between structure and agency and create a positive learning environment. Through their analysis, Getting Smart's researchers surveyed teachers from 11 different countries who have utilized Minecraft as a learning tool within their classroom to determine what impact the game had on the students' learning experience. The authors found that nearly $98 \%$ of teachers surveyed reported 
problem-solving as the primary skill developed by using Minecraft lesson plans and through extracurricular play. They also cited creativity $(95.5 \%)$ and critical thinking $(93.3 \%)$ as other primary SEL skills learned through the process, (Getting Smart Research Center, p. 10-11). Additionally, these skills do not stop at end of the lesson plan or the game of Minecraft, they continue to be pertinent in other social settings. They increase one's understanding of social and civic interaction, as well as an individual's access to various sources of knowledge and values pertaining to everyday life. In case studies from other schools around the world, the authors find direct examples of Minecraft continuing this facilitation of norms and skills outside of the classroom. For example, in the Bryant Montesorri School in Tacoma, Washington, students have access to an afterschool Minecraft club that has had noticeable impacts on their kids' socialization, empathy and personal connection others, (Getting Smart Research Center, p. 1415). These students are learning the importance of social connection and bonding, working with your fellow student, co-worker, etc. to achieve larger goals and respect one another in the process. Minecraft, through its insertion into lesson plans, can and does positively influence the overall disposition of students towards cooperation, problem-solving and empathetic feelings. This, in turn, creates a positive habitus within the virtual social field of Minecraft that carries over into school seamlessly.

Now that we understand the positive relationship that video games, specifically, Minecraft, can have with the learning process, let's shift attention to their relationship with civic engagement and urban planning. A study from Amanda Lenhart and her co-authors at Pew Research Center helps us to understand the influence that video games can have on our inclination towards civic participation. According to their analysis, the level of video game play in a teen is directly correlated to their willingness to participate in civic engagement. They find 
that of teens that play video games, $75 \%$ of them do so cooperatively with friends in-person or online, (Lenhart et. al., p. 26-28). Additionally, the cooperative games being played have a number of positive attributes that promote civic engagement like helping/guiding fellow players, exploration of social issues, and group identification and support systems through guilds and clubs. Lenhart and her co-authors' data show that those players who engaged in cooperative games with some or all of these features recorded the highest likelihood of engaging in civic and political behavior in the real world. One of the most influential factors in this process is a player's participation in online forums pertaining to their game of choice. Those that used the forums showed a $15 \%$ increase in civic engagement. Among teens who engaged in the highest civic gaming experiences like using forums or helping other players, according to Lenhart and her co-authors, $70 \%$ of them go online to get information about politics or current events. Additionally, they found that $70 \%$ have raised money for a charity in the year prior to the survey. Comparatively, $61 \%$ of the players exposed to the most civic gaming experiences expressed an interest in politics as opposed to $41 \%$ of the players exposed to the least, (Lenhart et. al., p. $40-$ 44). This data clearly highlights not only the positive social features associated with playing video games, but also how that socialization directly increases the player's willingness to be engaged in civil and political society. It exposes players to new ideas, values, and decisionmaking strategies. Video games can also provide players with a better understanding of how social and political systems are built and how they maintain their structure through critical thinking, cooperation and problem-solving. To add further support to this argument, Dmitri Williams' article, Groups and Goblins, provides us with significant evidence of this same phenomenon. Through his analysis, Williams finds evidence that playing video games is correlated with an overall increase in players' willingness to engage in civic participation like 
signing petitions, volunteering and even just increasing their general political awareness, (Williams, p. 659-664). Again, this data provided displays a clear and positive relationship between playing video games and an individual's willingness to engage in civic participation. Video games like Minecraft can also provide an avenue for direct civic engagement and urban planning, which I will discuss next.

In their study, Artan Rexhepi and his co-authors provide extensive evidence of utilizing Minecraft as an effective medium for citizens to be actively involved in the process of urban planning and development. The study uses 39 participants, all who were tasked with coming up with new and innovative design ideas for the "Former Marketplace" in Prishtina, Kosovo. Following a few days of work, the design ideas were collected, which included some useful and creative ideas like speed bumps, a clear roundabout, a climbing wall, creative art installations, and recreational facilities. What is fascinating about this study is that it actually created real world change to the city itself, as many of these features were used in the final design, (Rexhepi et. al., p. 52-54). The inclusion of Minecraft had clear, positive impact on another social environment outside of the virtual context, but it also facilitated a shift in how citizens of Prishtina viewed civic engagement. The authors concluded that, "The results of the conducted survey (figures 4 and 5) show that participants appreciated the initiative at all levels, including concept, organization, creativity, and community engagement. The participants had a clear understanding regarding the requirements for the project site through the presentations, site visit, community consultation. and own ideas and suggestions. Minecraft gave them the opportunity for a simple and unique way of expressing their ideas and transforming them into virtual elements, through a consolidated teamwork," (Rexhepi et. al., p. 54-56). Additionally, Fanny von Heland and her co-authors utilized Minecraft in a similar study to the previous one, only this 
time it is applied to an urban planning effort in Kirtipur, Nepal. There were 37 participants, all of which were given two days to come up with concept designs, built entirely within the virtual world of Minecraft, as possible plans for a public space within a local park. This study also took a number of ideas from the public, many of which were teens and young adults, and implemented them into the design. Following the completion of the study, participants were asked about their experience with this form of civic engagement, with most strongly agreeing that the process provided them with increased knowledge of other people's needs, helped produce meaningful dialogue, and improved confidence communicating viewpoints and speaking in large groups, (von Heland et. al., p. 5-11). These studies clearly exemplify the wide-reaching influence that the video game world, and the habitus it produces, has on other social environments. It facilitates more effective learning environments as well as easier paths to civic engagement, all while teaching us positive socialization and problem-solving/critical thinking skills.

\section{Conclusion}

This chapter set out to prove that video games not only positively impact our socialization and cognitive development, but also that those skills can be converted and utilized in other important social settings in our lives. Prior to breaking down these components, a theoretical framework for understanding video games had to established. We walked through Bourdieu's theory of practice, specifically how both individual and collective habitus influences our norms and dispositions within a social setting. Next, social constructivism and its breakdown of norm production and diffusion was incorporated into our theoretical position. Both these gave us a more grounded understanding of how our social norms and behaviors come to be and how both our own individual agency and the structure of the social setting influences them. 
Following this review of our theoretical background, the traditional understandings of both the positive and negative habitus of video games were broken down by analyzing the prevailing literature surrounding them. in the case of the negative habitus, authors like Craig Anderson have posited for years that video games caused aggression, antisocial behaviors and addictive tendencies. Looking into the positive side of the gaming culture, we see many of these traditional studies lack the knowledge regarding the variety and depth of the pool of video games in the current climate. More contemporary research explains how these emerging game genres and designs provide a host of cognitive, social and anti-aggression benefits with the incorporation of cooperative and critical thinking aspects.

In the analysis section of this chapter, we broke down first the negative, toxic, and abusive culture surrounding video games. Through this discussion, we pointed out examples of the active steps both game developers, and the community at large are taking to eradicate toxicity within the game. This process has proven to be quite effective in curtailing toxic behavior, but it is only one part of the process. The second portion of this analysis section looked at how games are actively promoting positive norms and values, through systematic changes to the game, rewards and commendations for good behavior and even giving more control to the players to regulate bad behavior in game. The analysis section closed with how these positive norms, values and dispositions are easily converted into social spaces, making us more efficient and successful in those settings. Overall, this chapter has proven the value of video games in producing, reproducing, and converting positive norms and dispositions that facilitate the development of a broad and positive habitus in a variety of settings. 


\section{CHAPTER II: STRUCTURE OF THE VIRTUAL FIELD \\ Introduction}

In chapter one we focused on how video games, as an emerging form of socialization and norm production, facilitated the development of positive norms and dispositions in players that had noticeable impacts both in-game and in other social settings like school, work and civic engagement. We briefly highlighted the importance of the setting and structure of the virtual environment in the previous chapter but did not give an in-depth analysis of the features of game design that help create what I call the virtual social field of video games. In this chapter I will breakdown how video games help create and stabilize an environment for interaction in-game and how the positive social engagement, along with norms and values produced through this socialization, can be influential in other environments as well.

The first section of this chapter will consist of a review of the prevailing literature that provides the theoretical support for this argument. To begin, I will break down Pierre Bourdieu's conception of the field from his Theory of Practice, looking at how social fields are formed.

Next, I will show how the internet and technological advancement has shifted our understanding of the public sphere, and how the immersive and interactive nature of video games has impacted social interaction in these virtual settings. This portion of the literature review will also find theoretical support in the concept of social constructivism, as it helps explain the importance of both the agent and the structure in the process of norm production and the diffusion of these norms. Both of these theoretical approaches will help set the framework for analyzing video games as a new form of social sphere.

Following this application of Bourdieu's conception of the field and constructivism to this analysis, an equally important theoretical approach must be incorporated to better understand 
the immersion and interactivity that is essential for effective socialization and development in video games. Flow Theory, a term coined by Mihaly Csikszentmihalyi, refers to, "the feeling of complete and energized focus in an activity, with a high level of enjoyment and fulfillment," (Chen, p. 2). Flow can manifest in a variety of ways, but most often occurs during activities we find immense enjoyment in (i.e. sports, arts \& crafts, even work) and helps to make the individual more efficient and effective at completing the task. Experiencing Flow requires a number of factors like clear objectives, consistent feedback, and an effective reward/punishment system, all of which are prevalent in video games. The immersion and interactive design associated with video games clearly falls under the umbrella of flow theory and its effects, which in turn makes us better at completing tasks, retaining the skills and information provided during the task, and creating an overall more enjoyable environment for the participant.

After I have established the important theoretical foundations for this chapter, I divide the analysis section into two parts: the first section will look directly at the eSports community and determine how and why it facilitates positive social interaction and development of important social and cognitive skills. The eSports community provides a variety of ways to be involved, ranging from an active participant to a spectator of the professional leagues to even being part of the governing body designed to regulate the competitive environment. Individuals involved in the eSports community learn important skills associated with cooperation like competition and teamwork, and cognitive skills that help with our ability to process information and apply it to other contexts. This section will use the Elo Ranking System and the TrueSkill Ranking System as its primary points of analysis, as they provide the best possible formula for measuring players' skill levels. Both of these systems will be adequately explained and contrasted against one another to determine what the overarching positive attributes associated with competitive 
ranking systems are and what the impact of their structure has one the development of positive norms and values.

The second section of analysis will investigate the importance of MMORPGs (Massive Multiplayer Online Role-Playing Games) like WoW (World of Warcraft) in providing social and civic engagement for its player base, as well as opportunities to develop positive social and cognitive skills in its players. These games provide a player with the tools to design an avatar for the game that is exactly to their liking in terms of appearance and skill set. These games require constant participation to make sure the in-game avatar is leveling up and maintaining the individual's in-game status with other players. Additionally, games like WoW offer social communities called guilds in which players can interact with others on their server. Guilds offer players the opportunity to work together to complete quests \& raids, as well as trade resources and take part in a number of civic and political forms of engagement. There will be two primary examples analyzed in this section that highlights the social and civic nature of WoW. The first will look at how the LFG (Looking For Group) system has had a dramatic impact on the social features of WoW, both positive and negative. The second will look at a specific in-game example, the Corrupted Blood Event, which highlights the strength and consistency of the community bonds established in WoW. This in-game event also provides us with additional features that mimic a variety of issues related to the COVID-19 pandemic, namely lockdown strategies and individual's behaviors that can both facilitate or stifle the spread of the virus. It is through these two sections of analysis (i.e. the eSports community and the MMORPG community) that we will clearly display the positive impact that the structure and design of video games has on our socialization and development of positive norms and values. In the following 
section we will begin our study by breaking down the supportive concepts of Bourdieu's theory of practice and social constructivism.

\section{Literature Review}

\section{Importance of the Social Field's Structure}

The beginning of Chapter One provided us with a solid framework for understanding and applying Bourdieu's Theory of Practice to the context of video games. the previous chapter placed a heavy emphasis on the concept of habitus and the role it plays in producing our norms and practices. Equally important to this process is the physical, or virtual, setting of the social engagement, which Bourdieu calls the field. In the context of video games, a true physical space is not available but the structure and design of the virtual environment mimics real-world social settings. This, in turn, makes it equally essential in establishing the parameters for social interaction. The field can be described as "a system of objective relations of power between social positions which are limited to a system of objective relations between symbolic points," (Mahar et. al., p. 8). What is most important for understanding the field for Bourdieu is that it is not a preestablished environment that is static and restrictive. Rather it is a constantly shifting dynamic setting that is produced by the constant struggle between actors interacting with one another. For clarification, the field is an embodiment of social interaction. The overarching social space, which refers to the general conception of the social world, comprised of multiple fields that have relation to one another and contain some points of contact. The social space is formulated and standardized by the interconnectedness between varying social fields, which helps to establish our cultural norms and dispositions while creating diversity in social interactions that take place. It is this process of building to a concise and all-encompassing social environment that makes the field so essential to understanding social practices. 
In Distinction, Bourdieu outlines the influence that the social field has, not only our social interactions, but our tastes and dispositions within that interaction. According to Bourdieu, "In the cultural market, the matching of supply and demand is... the result of the objective orchestration of two relatively independent logics, that of the fields of production and that of the fields of consumption," and, "thus the tastes actually realized depend on the state of the system of goods offered; every change in the system of goods induces a change in tastes," (Bourdieu, Distinction, p. 230-231). Now why does this matter in relation to virtual field of video games? First and foremost, this conception of the mutually supportive relationship between the fields of production and consumption help adequately explain the current relationship between video game developers and their player base. The community surrounding popular video games have, in recent years, become huge decision makers in the development and management of the game itself through forums, support websites and even direct communication with developers. Players express their concerns about broken game mechanics, suggestions for new game modes or missions, and even changes to aesthetic features, all of which facilitate a change in the overall tastes and dispositions of the player base. The gaming community (field of consumption), and the developers (field of production) are therefore interrelated with one another, creating an environment that requires the cooperation of both fields to operate effectively. Additionally, video games, as form of a social field, have far more flexibility when it comes to assigning value to actions in-game, skills learned through playing, etc. than other fields normally would. The game's designers have full control over the mechanical and structural aspects of the game that guides players towards different objectives and, in turn, rewards for completing them. The developers of these games, through the design of the setting and mechanics, set the value for everything earned and learned within the game, making the structure essential in understanding 
the process of norm production and diffusion. On the other side, the game's themselves would fail without the incorporation of the cultural tastes and dispositions of the player base guiding the design of the game. The analysis section will break down this concept with specific examples of how both Overwatch and WoW are responsive to, and in some ways dictate, the norms, tastes and dispositions of their communities while still finding ways to meet to meet their needs.

Before moving on to discuss the importance and influence of Flow Theory, I must first incorporate social constructivism into this analysis, as it provides a framework for understanding the relationship between agency and structure. Social constructivism, according to Risse's article, adheres to a "logic of appropriateness," something I mentioned briefly in the previous chapter. This system of logic, in-line with Bourdieu's logic of practice, explains that an individual's identity and actions shift with the social setting they are currently in, (Risse, p. 3). The major difference between the two lies in the fact that the logic of appropriateness focuses on rule-guided behavior as opposed to the logic of practice being dependent on the relationship between our personal norms/dispositions and the rules of the social environment. That being said, both forms of logic heavily emphasize the importance of having varied approaches to social interaction based on the current environment (i.e. different situations call for different demeanors and actions). The actor makes adjustments to their behavior and dispositions depending on what the environment deems as acceptable and is supported it, (Risse, p. 5-6). For example, the norms and behaviors that an individual understands as acceptable in a setting like school or work is vastly different from playing a sport, socializing in a bar, or even talking with someone outside of their normal social circle. Humans are extremely intuitive and make these adjustments to facilitate a positive situation by noticing and adhering to the shared norms and values. This concept is the same with video games but goes a step further in that our dispositions can change 
on a game-to-game basis (i.e. switching from FPS, first person shooter, game like Overwatch to an open world MMORPG like WoW). A player's disposition and understanding of the social environment can even change in-between matches or quests, as the objectives, team dynamics and possible rewards are all subject to change. There are overlapping skills, tools and norms that are applicable to many different game genres and platforms, which will be discussed at length in the analysis section as well. Regardless of this however, the structure and design of video games plays an enormous role in the development and stabilization of norms and dispositions within virtual social fields. The two most influential aspects of this design, immersion and interactivity, are integrally linked to the production and usage of the sensation of Flow, which will be the focus of the following section.

\section{Flow Theory, Immersion and Interactivity}

The sensation of Flow is a well-documented phenomenon in social science research and lends valuable insight into goal orientation, socialization and overall human behavior. According to Michailidis and his co-authors, Csikszentmihalyi outlines nine major features associated with flow. These are balance between individual skill and demands of the activity, merging of action and awareness, clear goals, immediate and unambiguous feedback, concentration on the task, perceived control over the activity, loss of self-reflection, distorted perception of time, and intrinsic motivation toward an activity, (Michailidis et. al., p. 2). As these classifications show, understanding and analyzing the presence of flow is challenging and complex and can be broken down even further. In relation to our topic of video games, flow theory finds numerous overlaps with the concepts of both immersion and interactivity. Let's first discuss the interactive nature of video games and how it correlates with producing flow. 
Mihaly Csikszentmihalyi points out in his article that, "flow generally occurs when a person is doing his or her favorite activity... very rarely do people report flow in a passive leisure activity, such as watching television or relaxing," (Csikszentmihalyi, p. 2). These activities therefore must involve active engagement and video games are great example of this as they, unlike many other forms of modern technology, require hands on decision making, problem solving, etc., to appreciate and enjoy the experience. One of the major issues that video game developers have had to deal with is the first feature of flow, the balance between the participant's skill level and the challenge of the objectives. As Jenova Chen points out, if the challenge is too high for the player, the activity becomes overwhelming and induces anxiety; if the activity is unable to engage the player, they experience boredom and quickly lose interest in the activity. One of the major ways that games adjust to this fact is by scaling difficultly levels for players to allow for a variety of skill levels to enjoy the content provided, (Chen, p. 3-4). Most story mode video games offer a difficulty scale ranging from beginner to some form of expert and the difference between the top and bottom skill brackets is quite significant in most cases. In the analysis section focused on WoW, I will look at how Blizzard Games, the developers of WoW, have worked to create a balanced environment for players of all skill levels with a vast array of quests, dungeons, raids, and player vs. player $(\mathrm{PvP})$ options that contain a range of difficulty options.

Along with the balance between skill and challenge, the interactive nature of video games is also dependent on the clear goals, unambiguous feedback, and a reward/punishment system. According to Sanjamsai and Phukao, these features can be classified as "cognitive flow" and state that it helps us to recognize the advantages associated with playing video games, along with the time spent playing, (Sanjamsai \& Phukao, p. 178-180). Clear goals, feedback and 
reward/punishment systems are the foundations of any enjoyable activity and are essential for any video game to operate effectively. This process is facilitated by reward systems in games which, according to Wang and Sun's article, fall into eight major categories. The first four, score systems, experience points (XP), item granting systems, and resource systems, all fall under the classification of visible or usable rewards for the player. In the case of the score system and XP, players earn in game points or currency that help advance their prestige in the game or their avatar's level. Resource and item granting systems help players collect and utilize in-game items, either as resources for the gameplay itself, or as cosmetic aspects of the character. The last four, achievement system, feedback messages, plot animation/cut scenes, and unlocking mechanisms, represent effective feedback for the player. Achievement systems notify players of their completion of objectives and provides them with signifiers on their account to notify other players of the achievement. Plot animations and cut scenes provide structure and direction for the narrative of the game, keeping the player both on track and engaged in the game's content. The unlocking mechanisms serve a similar purpose to achievement systems but also have the added social benefit of being one of a handful of players to complete the locked task. These reward systems are measured based on four major characteristics, social value, impact on gameplay, suitability of the reward (i.e. how much is it motivating the player), and time required to earn/receive an award, (Wang \& Sun, p. 4-6). These measurement tools can help game developers determine the effectiveness of their reward systems and make adjustments if necessary. These systems help to avoid player burnout by modify the individual's emotions through rewards and establishes higher challenges for players to work towards. Now that we understand the importance of interactivity and how it relates to Flow, let's turn to a discussion of the concept of immersion. 
Immersion involves the complete investment of the individual into an activity, engraining themselves into the activity and the environment it takes place in. This concept is essential for understanding flow for a number of reasons. First and foremost, immersion helps facilitate the production of the other form of flow from Sanjamsai and Phukao, emotional flow. According to them, emotional flow is composed of action-awareness merging, concentration on the task at hand, sense of control, loss of consciousness, and time transformation, (Sanjamsai \& Phukao, p. 178-180). These features clearly relate to an immersive experience and highlight the possible positives, and negatives associated with video game play. One of the major positive effects of engaging in an immersive experience is the impact it can have on our place identity. According to Bonaiuto et. al., place identity refers to the features of a person's identity that are related to a person's relation to their specific environment or setting, (Bonaiuto et. al., par. 3). Place identity helps us to form our perception and dispositions towards our environment and the individuals we interact with in that setting. This supports Bourdieu's explanation of the role of the structure in establishing our norms and practices. They further find that the most likely correlation between an individual having an immersive flow experience and establishing their place identity is when skill and challenge levels of the activity are the highest, (Bonaiuto et. al., par. 23-26). This again speaks to the importance of immersion into an activity when trying to facilitate the production of flow.

The immersive nature of flow experiences can also be described as "blissful productivity," a termed coined by Jane McGonigal in her book, Reality is Broken. McGonigal outlines blissful productivity as a sense of being so deeply immersed in work that it produces immediate and obvious results. McGonigal's research is heavily focused on WoW and the positive attributes that these open world virtual environments can provide. These games, as she 
points out, are designed so that the players have opportunities for constant improvement of their playable avatar by leveling up gear and skills along with challenging more difficult opponents. One of the major reasons for why these games are so successful at immersing their player base is the required teamwork and cooperation for many of the quests and missions available to players, (McGonigal, p. 55-62). This forces players to not only be invested in their own actions and decisions, but also their teammates and other players within the server. To be effective in WoW, you have to be willing to be a part of the community, interact with others and work towards shared goals, all of which speak to the game's immersive nature. Immersion is not always a positive, however, as it can lead to addiction and disconnection from the outside world. In his book, Enjoying It: Candy Crush and Capitalism, Alfie Bown describes what he calls distracting games, which are quite different from MMORPGs but still have the opportunity for a number of negative outcomes. Most games that fall under this classification would be games like Candy Crush, Angry Birds, etc.; games that are designed for pure enjoyment and for filling spaces of boredom in our lives. Bown argues that while these games help to distract us from stress and frustration in our lives, they also work to pull our focus from important tasks and decisions that require our attention, (Bown, p. 26-28). These games are a way to find fulfillment in our lives when our other social environments like school or work are not doing so, but because of this the importance that we put on those other activities diminishes over time.

Before moving forward into the analysis of the primary examples in this chapter, we must first relate flow theory and its features to the overarching theory of practice utilized in this thesis. The main point of relation between these two theories is in the importance of the field in establishing our behaviors and interactions with others. As I mentioned above, the sensation of flow is achieved through nine important factors like clear and challenging objectives, feedback 
and reward systems. These are all based on the formation of the social setting in which the actor is operating, which must not only be structurally sound, but also incorporate the culture and norms of the actors present in the setting. The tasks outlined for the player in a video game, for example, will not keep the player engaged and interested if they do incorporate values and skills that are important to the player like cooperation, critical thinking and character development. The inclusion of cultural features into the tasks not only further facilitates the production of flow; it also provides the proper social environment for creating the practice that Bourdieu's theory outlines. Player's tastes and consumption patterns largely dictate how and why we play certain video games, which as I mentioned above, is facilitated by how engaging and challenging the games themselves are. Therefore, Bourdieu's theory of practice finds important overlaps with flow theory and they provide support to one another in the proceeding sections of analysis.

Now that Flow theory, immersion and interactivity have been analyzed and incorporated into our discussion of the virtual field of video games, we can now turn our attention to applying our theoretical position to some actual examples of video games and their structure. In the first analysis section, I will look at how the eSports community has worked to create a positive social environment for it player base by making adjustments to the structure and design of the game as well as promoting social engagement through a number of innovative features like Role Queue system we mentioned in chapter one. Following this, I will examine the structural features of the popular MMORPG, World of Warcraft and see how they have shifted to promote positive social and civic engagement in its player base. 


\section{Virtual Social Field of Video Games}

\section{Positive Impacts of the Competitive Gaming Environment}

The development of the video game industry, and the community that it spawned, has been complex yet flexible. Video games have expanded into countless genres, design styles, etc. but one thing has maintained as an essential aspect of nearly every video game since the days of Pong, competition and the opportunity to come out the victor. Competition is not always the primary goal of the game; many solo story mode games have little no direct competition between players. However, these game then in turn pit the player against themselves, working to beat their best score and feel that sensation of accomplishment that comes with success. Competition, whether it be against other players, yourself, or in-game CPUs, is what makes video games enjoyable and makes players return to them. The competitive gaming, or eSports, community is an enhanced and direct example of the importance and value that competition has when it comes to playing video games.

Looking at eSports through the lens of flow, and to a larger extent Bourdieu's Theory of Practice, we understand that the structure developed by game designers, as well the cultural identity of the player base, work in tandem to facilitate an engaging and enjoyable gaming experience that. These, in turn, create positive norms and values in players, some of which are introduced in this field by players, that can produce flow and positive socialization in other setting as well. According to Yuri and Jung, making a well-developed eSport requires creating a "rich sensory experience that calls for layer upon layer of physically demanding action in order to be competitive in a high-performance game," (Yuri \& Jung, p. 643). So how do the game's developers help to facilitate not only a competitive environment, but also one that provides positive personal development, cooperation/teamwork, and even problem-solving and critical 
thinking skills? One of the major ways that games like Overwatch and Call of Duty have worked to promote the competitive aspects of their game is through a ranked, league play system. Ranked systems in video games are a relatively new phenomenon, rising in popularity in response to the support for eSports leagues and they mimic many of their key features. Most games like Overwatch have a professional eSports league that has high paid players on 12 different franchise teams and have unique rules, map sets, and styles of gameplay that are vastly different the average public match that a player loads into. This is intended to make the game more balanced and foster a more competitive environment that is based on the player's skill rather than the semi-uncontrollable aspects of the game like poor connection or overpowered weapons and gear. For instance, in the Call of Duty League, players are not allowed to use certain weapons, equipment, and character buffs, as they are deemed too unbalanced to be allowed into a competitive setting. The ranked systems in these games often copy and paste the ruleset for their respective professional league, as it helps new spectators pick up on the game's flow easier.

There have been a variety of ranking systems used throughout the development of these leagues, many of them using the systems popularized by chess tournaments in the $40 \mathrm{~s}$ and $50 \mathrm{~s}$. In this chapter however, I will only focus on two, as they are the most prominent, well known, and arguably the most effective at determining a player's skill level. The first of the two, the Elo System, was, as I mentioned in the previous chapter, was first developed by Arpad Elo in 1959 for the World Chess Federation. According to Ourania and their co-authors, the Elo System utilizes the Thurstone-Mosteller model to estimate the probability of individual game outcomes. In this system, players are assigned a score between 0 and 3,000 that changes over time based on the mean outcome of their performances, measured by wins, losses, and draws, (Ourania et. al., 
p. 3-4). This is system has become widely used in most modern multiplayer video games due to its simplicity and its ability to place players in the correct tier based on their performances. One major concern of game developers and the community at large, however, is the fact that this system may be too statistically geared, making it challenging to incorporate intangible factors that happen during games into the equation. To try and account for this, Microsoft's research team worked to develop a new and more intuitive formula for ranking players, the TrueSkill System. This will be the second system we will focus on in this section, as it has become the staple of most eSports ranking systems, replacing Elo in many cases. TrueSkill, in theory, is quite similar to Elo, with the major difference being it incorporates both initial score as well as previous and possible future scores, giving the most robust system possible. Ourania and her co-authors also point out that in team-based multiplayer games, the TrueSkill system estimates a player's ranking based on the average scores of their teammates, as well as their opponents and all of their individual performances, (Ourania et. al., p. 6-8). Essentially, what this means is that if you play well in a game, but your teammates do not, you will not be punished nearly as much as you would if they played well. Additionally, if the other team is clearly better and beats your team handedly, the game takes that into account when giving you your score.

Even though TrueSkill has become the staple of most competitive games, Elo has become the offhand term used when discussing one's rank in-game. This has also bred another new term as well, "Elo Hell," which refers to getting stuck in a specific rank tier and being unable to increase your position due to a number of factors like bad teammates or matchmaking issues. In his study, Daniel Espinosa wanted to determine if Elo Hell 
was actually real by looking at players current rankings compared to what his model projects their placement based on their true skill and performance. What he finds is that most ranking systems are actually quite accurate at placing players in their correct tier, with only around $2 \%$ of the 2500 "elite" players surveyed were found to be misplaced. Additionally, only around 3.5\% of the total 10,000 players surveyed experienced misplacement which speaks to efficacy and efficiency of these ranking systems. The author did mention the same issues that were raised in Ourania's piece that Elo is ineffective at incorporating teamwork and other intangible features into its analysis. When he did incorporate them, did find somewhat significant shifts in the survey population, as the percentage of the total population of players who were misplaced shifted from $3.5 \%$ to $10 \%$, (Espinosa, par. 13-19). There are a few ways that a player can make adjustment to increase their Elo and become a better player overall. Moncav suggests working on your communication, as both over and under communication can hinder the team's success. She also advises that players focus on their own individual performance not their teammates and take the time to practice and make themselves better, (Moncav, par. 10-11).

What is important to remember about this intensely competitive environment is that it is not for everyone, but those that invest themselves in it will find positive opportunities to train a variety of skills. In their article, Amelia Carbonie, Zixiu Guo and Michael Cahalane break down the eight major benefits and 4 major values that the individual's they interviewed associated with competitive eSports play. They pooled 19 professional League of Legends players from their Australian division and identified their major motivators as achievement, socialization, immersion, self-improvement and competition. Through the interview process, the authors established that commitment and cooperation were the most important benefits, each having all 19 respondents. Initiative and tangible rewards were the next highest (18 respondents each), and 
Emotional self-regulation (17), personal growth (17), self-esteem (16), and communication (15) rounding out the benefits. In terms of the values they found important, all 19 agreed that hedonism (i.e. emotions related to pleasure and enjoyment) was the number one value. The other three included power (16), purpose in life (16), and achievement (15), (Carbonie et. al., p. 6-7). The responses received by Carbonie and her co-authors gives grounded support to the argument that eSports has positive influence on its player base through the promotion of positive social and civic values. Overwatch has tried to incorporate more direct experiences of these positive values, both through their Role Queue system and the post-match accommodation system that I mentioned in the previous chapter. These facilitate better team dynamics and give players direct feedback on their performance that may not be directly related to their Elo score. The competitive eSports environment has clearly given a variety of positive benefits and values to some of League of Legends top players, so we can posit that those same benefits and values can be experienced by average players as well.

Before moving on to the second section of analysis, we must explain how these positive benefits and values are not only helpful for the players in-game, but also for the average players, spectators and the governing bodies of the industry. Breaking down the scope of these benefits and values helps to highlight the levels of social and civic engagement involved in advancing eSports leagues. For the purposes of this thesis, I will assume that eSports fall under the umbrella of a sporting event and therefore carries with it a number of the same positives that traditional sports would. Obviously, we are not assuming that the physical and health benefits are comparable, rather we will find overlap in the important social and civic values like cooperation and problem solving. With this 
in mind, we turn to Ritz's analysis of sports participation and civic engagement. In his study, Ritz finds that there is a direct and positive correlation between sports participation as an adolescent and volunteering in young adulthood. Additionally, he found that sports participation was actually more influential than church attendance or level of education on a young adult's willingness to volunteer, (Ritz, p. 20-26). The author credited much of these results on the factors of sports associated with socialization, cooperation, and concern for others, all of which can be directly applied to the context of eSports participation. It also highlights that the positives associated with participating in a sports event of any kind does not require regular participation. An individual can gain those possible benefits for civic engagement from simply playing as a child and again, this logic can be applied to video games as well. Benefits and values associated with competitive eSports play can be ascertained by individuals who are not active participants and those that are not are equally essential in facilitating this positive environment. Seo and Jung make a point that to achieve the practice that the game developers want, the game itself must involve "a nexus of understandings, tools, skills and competencies, which social actors integrate dynamically through their routinised performances," (Seo \& Jung, p. 639). Carbonie and her coauthors echo this point by stating that eSports consist of complex and mutually supportive relationships between professional players, sponsors, fans, and eSports society as a whole, (Carbonie et. al., p. 3-4). As I said before, this system is complex yet flexible, considering that even though the eSports environment is demanding, there are a variety of ways to be a part of the community that do not require an individual to be a premier player. Spectating eSports events, both in-person and through streaming services like Twitch, has had a profound impact on the growth of the industry and has allowed for new viewers to learn tactics, lingo, etc. without having to even play the game. Think of it like a casual viewer of the NFL; they may have never 
played football before but thanks to commentators and actively watching the sport, they learn more about and feel engaged in the game. Additionally, the growth of the governing bodies of the eSports leagues is fascinating and essential for the development of this industry. By providing more rules and regulations, eSports leagues become more professional and institutionalized. This, in turn, makes it easier to reach a larger audience, earn more revenue and expand the league's size. Seo and Jung do point out that for the governing bodies to be effective, they must immerse themselves within the community, learning the intricate skills, tools, and tactics of the game to be better prepared to make adjustments, (Seo \& Jung, p. 646-648). These governing bodies also have an added and unintended benefit of teaching those involved in the community about proper decision making in governance, as well as what makes good public policy. These can operate, in theory, like small scale democracies, as the players and spectators often become integrally involved in this governing process.

Now that I have broken down the diverse and intricate system of eSports leagues, we can move on to our second section of analysis, the socialization and civic benefits of the MMORPG World of Warcraft. This next section will look at how, through the medium of an individual's in-game avatar, players learn positive socialization skills as well as varying ways to participate in civic engagement, all within the context of the virtual world of Azeroth. I will also look at how Blizzard Games has worked to promote socialization, as well as how the social dynamics of the game have changed over time and what that means for the opportunities for players. The section will close with a look at some specific examples of both positive socialization and the use of civic engagement in player-to-player interaction. 


\section{Social and Civic Value of Playing MMORPGs}

Shifting our focus now, let's turn to analyzing the social and civic value of the MMORPG World of Warcraft. WoW is set on a fictional continent known as Azeroth that has a catalogue of character species, skill attributes, play styles and social groups available to players. In WoW there are two major "factions" within the game, the Alliance and the Horde. The Alliance consists of five races that a player can select from when creating their avatar which are humans, dwarves, night elves, gnomes and draenei. The Horde is the other available faction and also has five races which are orcs, undead, tauren, trolls and blood elves. Each of these different races have unique skill sets, specified gear, and in some cases, unique rewards for that character build that are unavailable to other races. While these do seem like diverse options, many players have complained that these races are limited and lack actual diversity in terms of representation, (Mosley, p. 57-58). The two alliances are set as opposing sides of the PvP (player vs. player) conflicts but faction selection impacts PvE (player vs. environment) aspects as well. PvP events consist of competition between and against other players, most often taking place in the arena, battlegrounds or while exploring the map. PvE involves cooperation with other players to complete complex and challenging tasks against NPCs (Non-playable characters). In the case of PvP events, Mosley finds that around $48 \%$ of Horde players on are PvP servers, highlighting a correlation between character selection and server selection and that Horde players are, on average, more aggressive and/or interested in the competition aspects of the game. Additionally, when looking at the effects of personality traits, Mosley finds that there is little to no data to support that avatar or faction selection is dependent on personality traits. However, he did find that players who reported playing WoW for joy of completing group and co-op objectives 
showed high scores in extroversion, agreeableness, intellect and tolerance, (Mosley, p. 59-62).

This data clearly highlights the positive impact that cooperation and teamwork within the context of a virtual environment can have on the player. The team-based objectives in the game are correlated with positive social norms that can in turn be utilized outside of this context as well.

Before continuing to discuss these social and possible civic benefits, it's important to discuss the process of avatar creation and how its immersive nature influences the player's gaming experience. According to Lau's article, avatars are virtual versions of the player, representing the complex processes and ongoing practices involved in constituting the individual and establishing an identity in virtual worlds. He references Merleau-Ponty's theory of body schema, which posits that certain objects or tools related to our body's schema (i.e. a blind person's walking stick), function not as tools needed to mediate our lives, but rather as incorporated extensions our physical body, (Lau, p. 381-382). Video game avatars operate in a very similar way, embodying the cognitive instructions given to our body. In essence, utilizing a joystick to run becomes as integrally associated with the act of running as telling are legs to move is. This process of making the controller and the in-game avatar an extension of the decisions and actions of the player give us some more insight to the intensity of the possible immersion into the game itself. It blurs the lines between reality and the virtual world, which is something Nick Yee discusses at length in his article, Labor of Fun.

In his research, Yee finds that in many examples of MMORPGs, the player's experience can be referred to a "second job," in that the games themselves are, "inherently work platforms that train us to become better game workers," (Yee, p. 1). These games train us to be more efficient and effective at completing tasks set before us, 
much in-line with the standard expectations of the average job in the physical world. Many of these games, as Yee points out, not only provide a variety of jobs or roles within the game that players can engage in, but also a complex and expansive in-game economies that in some cases, can be compared to the financial system in the real world. These economies are also almost always player-driven, meaning that the NPCs cannot help to facilitate the production of resources or the trading of them, making the use of socialization and civic engagement exponentially more important. These games also struggle with pulling people into the game, as they clearly exemplify a number of time and effort barriers not unlike real work. To combat this, many developers have work to disguise the "work" aspects of the game, making a more enjoyable and rewarding and environment and avoiding burnout, (Yee, p. 2-4). Adjusting their game design to promote more enjoyable and engaging work shows that these games not only see the value of having their players experience flow, but also that it is necessary for an effective open world game like World of Warcraft.

Another, more recent, method that game developers are using to keep players immersed and invested in their virtual world is through the use of microtransactions. According to Zendle and their co-authors, there are two types of microtransaction, purely cosmetic and pay-to-win. Cosmetic microtransactions involve "any situation in which spending additional money leads to an aesthetic change within a game but no in-game advantage," (Zendle et. al., p. 2). Pay-to-win microtransactions refer to "any situation in which players are able to exchange real world money for something that increases their chance of in-game success," (Zendle et. al., 2-3). Due to largescale community backlash to pay-to-win microtransactions, most games try and avoid them at all costs, instead opting for the purely cosmetic options. These character skins, vehicle decals, etc., all serve an important purpose in these games of increasing a player's status and prestige in 
social space. An individual's position within the social hierarchy of any setting is an essential aspect of Bourdieu's theory of practice and it helps to explain the reasonings behind why actors present themselves in the way they do. In chapter three, I will go further in-depth on the development of in-game hierarchical structures and how they have impacted the player's experience. The in-game cosmetics have adopted exceedingly more complex and intricate designs, making players want them more and eventually leading to them to purchase. By owning these rare skins, players feel a sense of unique identity within the game that differentiates the players from others around them. This unique identity further immerses the player into the virtual environment in ways previously unexpected. As Zendle and their co-authors point out, the percentage of cosmetic microtransaction exposure for players has increased from $8.3 \%$ of all game experiences to over $85 \%$, (Zendle et. al., p. 7-9). Microtransactions are no longer a small side aspect of major video games, they are now the primary source of revenue for them and are valued at an all-time high by players in the community.

Now that we understand the role of avatars and cosmetic features in creating an immersive experience for players, we can now discuss how MMORPGs like WoW can positively impact our socialization and attempts at civic engagement. In their article, Zhang and Kaufman state that MMORPGs operate as "character-mediated social communities," (Zhang \& Kaufman, p. 151), which is facilitated through group and cooperative activities in game that are required to earn specific gear and level up in-game avatars. WoW has found an extremely effective way in promoting socialization and cooperation between players, which is their guild system. Guilds operate as essentially virtual social clubs that have the added benefit of helping players find other to complete 
team-based quests with. These social settings provide players with the potential for long-term bonding and allows for players to establish real social connection or a sense of accomplishment, both as an individual and as part of a group. What's fascinating about guild membership is that players tend to be quite loyal and consistent in their activity within guilds. According to Poor and Skoric's research, around 2/3rds of WoW's player based maintained their current guild membership over a three-year period, (Poor \& Skoric, p. 2269-2271). Loyalty to the close-knit community within the game is commendable and is in noticeable contrast to social groups in the non-virtual world. As Robert Putnam's book Bowling Alone tells us, social group membership is diminishing across the board in the US. Whether that be book clubs, neighborhood groups, or even bowling leagues, membership is at an all-time low for social groups. I believe that the stark difference between the two is due in large part to the immersive yet flexible opportunities available to players in these guilds. These guilds have an important role of being both a social environment for players to engage with one another, not unlike the social groups in the physical world. Additionally, they offer utility to the players as a setting for learning new tactics in-game, trading resources and building teams for completing tasks. This secondary role makes these guilds vastly different from the average social group, as the create economic and civic value for the player along with the inherent social value. The relationships you establish in these guilds have important social aspects associated with them, but they are equally as essential as places for economic transactions and skill development.

Another great facilitator of socialization within the world of Azeroth is the group finder system. This system, not unlike Overwatch's LFG system, provides players with a mechanism for seeking out other players to help complete quests, raids, etc. without having to already have social connections within the game. According to Rossi's research, some critics argue that the 
group finder system is actually diminishing the social capacity of WoW in that it removes the important character-to-character interaction out in the open world as well as removing the need for guilds entirely. Players wait in major hubs in the cities of Azeroth, spamming the group finder chat features hoping someone will accept their invitation to complete the objective. Rossi does argue however that this is an inaccurate way of the looking at the group finder system. Instead of seeing it as a hinderance to socialization, it should be looked at as a way to streamline the group creation process and minimizes unnecessary chat conversations that muddy up the chat $\log$, (Rossi, p. 3-7). When looking at these social interactions we see that the most common ways of communication within the game are group and private chats, making up around $56 \%$ of all socialization within the game. Additionally, Zhang and Kaufman point out that $76 \%$ of players noted establishing positive social relationships in the real world with players they met in-game and 39\% of players were willing to discuss personal information with in-game friends that they would not discuss with their real life friends, (Zhang \& Kaufman, p. 156-162). These socialization dynamics have a few important positive consequences, first of which is that the bonds made in-game persist outside of the context of the social world, making them valuable in other social contexts. Additionally, the fact that players were willing to share personal information with what essentially amounts to strangers proves that the immersive and interactive nature of these games make players feel comfortable to be themselves and express emotions they would not otherwise. This virtual setting lowers inhibitions of the players and gives them confidence to be themselves. I do want to note that Zhang and Kaufman's research did find some important challenges that may be present for MMORPG players. The two most likely challenges were diminished exercise 
habits, which around $21 \%$ of players reported experiencing, and noticeable fatigue after playing for a few hours, of which around 20\% agreed with, (Zhang \& Kaufman, p. 162-163). These are important to note and incorporate when analyzing the impacts of MMORPG play, but they are not drastic impacts on the individual's day to day lives.

To conclude this chapter, I would like to address the potential for civic engagement within the context of WoW. In his article Lau notes the potential new space for democratic interaction available in MMORPGs. As he points out, the unique combination of deeply immersive virtual worlds/real world technology and social and corporeal phenomena opens the possibility for a new space of democracy, (Lau, p. 383-385). As I mentioned above, Blizzard offers a variety of communication features in WoW, all of which fall under four basic categories of communication channels. These are General Chat, Trade, Local Defense, and Guild Recruitment, with the first being the most frequently used methods. These channels help guide players to their desired social setting and help find the players they need to complete tasks. These communication channels also provide a democratic discourse between players, as many players find themselves discussing and debating political issues all within the context of the world of Azeroth, (Lau, p. 386-388). One of the best examples of effective civic engagement within WoW would be the Corrupted Blood incident. According to Jennifer Ouellette's article, "In 2005, Blizzard Entertainment added a new dungeon called Zul'Gurub into World of Warcraft for highly advanced players, controlled by an 'end boss' named Hakkar. Hakkar was a blood god known as the Soulflayer, who had, among his arsenal of weapons, a 'debuff' spell called 'Corrupted Blood.' Infected players would suffer damage at regular repeating intervals, draining away their 'hit points' until their avatars exploded in a cloud of blood. The only cure was to kill Hakkar,' (Ouellette, par. 2). This debuff became an epidemic within the game as it had a number of 
unintended consequences. First and foremost, the developers had intended for the debuff to only impact players who are actively fighting Hakkar. The issue is that they did not anticipate players panicking when the debuff was activated and teleporting back to populated areas in the cities. This allowed for thousands of players to become infected almost instantly and for many lowlevel players, this meant immediate death as their character was not strong enough to withstand the damage. As Ouellette points out, the behavior of players and the responses of players to this disease were not unlike the response we've seen to the COVID-19 pandemic over the last year. One of the major ways in which they mirror one another is the willingness of individuals to knowingly and maliciously spread the infection with others as a way to create a type of herd immunity to it. What's important to note with the WoW Corrupted Blood debuff is that there was an unlimited amount of times a player could become infected, meaning that if they died, they would respawn and have the potential to be infected once again. Additionally, more empathetic players attempted to use their healing abilities to remove debuff, (Ouellette, par. 5-8). While this did represent good attempts at civic engagement between players, it actually allowed for the debuff to persist much longer than expected, as removing the debuff only freed up that player to possibly being infected again. Finally, as an end all response to stop the effects of the debuff, Blizzard decided to shut down all WoW servers a week after the outbreak, (Elker, par. 3). This, to me, seems like a pseudo-quarantine happening entirely within the context of a virtual world and it proved to be effective as the developers were able to find the source of the problem and remove the debuff from the game. This speaks not only to the efficacy of quarantines in any context, but also that these games facilitate effective and supportive forms of civic engagement for their player base. 


\section{Conclusion}

Now that we have properly analyzed the social and civic value of WoW, we can now review the arguments that have been made in the chapter. To begin, we established the importance of the field within the context of producing our norms and practices, based on Bourdieu's Theory of Practice and Social Constructivism. Following this breakdown, we applied Csikszentmihalyi's Flow Theory to the virtual field of video games, analyzing the immersive and interactive nature of them to establish how they facilitate proper norms and values.

In our analysis section, we broke down first the positive social attributes associated with the eSports community. We looked at how playing competitively can positively impact an individual's cooperation, problem-solving, and critical thinking skills. The use of Elo and other ranking systems creates a competitive and challenging environment, pushing players to get better at the game and learn how to cooperate better. Additionally, we discussed how the expansion of the eSports community has opened up to incorporate spectators, average players, and governing bodies, all of which help to develop a more diverse and intricate player population. The governing bodies also help to teach players about the important of institutionalized organizations and how effective civic engagement can be at making the social setting better.

In the second section of analysis, we investigated the social and civic aspects of the MMORPG World of Warcraft. In this section we look at how the avatar creation and development in these games creates positive personal development and allows for players to establish and identity within the context of the virtual world of Azeroth. These games also create positive social interaction through the use of social groups called guilds, in which players become members and work together to accomplish common objectives and build the strength of the group, and a Group Finder system. Finally, we looked at how those social features can spawn 
positive, and sometimes negative, civic engagement through the use of chat logs. This chapter has clearly highlighted how the social aspects and the structure of both eSports titles and MMORPGs like WoW has facilitated positive social interaction and opportunities for civic engagement. 


\section{CHAPTER III: HIERARCHY AND CLASS STRUCTURE IN VIDEO GAMES Introduction}

In the preceding chapters, I established both the overarching habitus of the video game community and how it influences the development of positive social, cultural and political norms in its player base. Additionally, I broke down the importance of the social setting - the virtual field of video games - as an integral aspect in the production of those norms and practices, as well as the conversion of them into other social settings. In the third chapter of this thesis, I will look at how these two features, an individual/collective habitus and the social field, facilitate the production and stabilization of a social hierarchy within the context of the video game world.

In a review of the existing literature, I will first reestablish the pertinent aspects of Pierre Bourdieu's theory of practice, most importantly his discussion of class position and social hierarchy. Additionally, as effort to better conceptualize hierarchical structures, this portion of the literature review will also include an analysis of more well-defined examples of social and political hierarchy. The literature surrounding class structure and social position will help to give a frame of reference for when I establish the commonalities between traditional social spaces and the emerging virtual world of video games.

After I have laid the foundations of a general understanding of social and political hierarchy, the remaining portion of this literature review will focus on what a social structure looks like in the context of video games. The most common forms of social organizations within video games happens in MMORPGs like WoW in the form of guilds or factions, which will be briefly discussed in the review but more critically examined in the analysis section. These guilds provide a variety of opportunities for socialization, teambuilding and even civic engagement, making them excellent areas of exploration. The other most likely area for hierarchal structures 
to arise is in the form of competitive ranking systems like the Elo and TrueSkill system discussed in Chapter 2. I will also establish the foundational aspects of social score and commendation features in FPS games like Call of Duty and Overwatch. These features, while not directly influencing your Elo rank, help highlight your cooperation or leadership abilities to your team and hopefully encourage better team dynamics for players. This section will also require additional analysis of Jane McGonigal's book, Reality is Broken, as her chapter on social connections and bonds within video games helps us to better understand the development of social position and class structure in-game.

After providing an extensive review of the pertinent literature, I will then shift my focus to analyzing the two primary areas of social structure development in video games. This section will be defined by the establishment of social, community based hierarchal systems that help to facilitate problem solving skills, critical thinking, and cooperation, as well as educational opportunities regarding economic transactions and player development. One of the major examples of this, as I mentioned above, is the formation of guilds in MMORPGs. These guilds provide a setting that operates in many ways like a traditional social structure, with a leadership hierarchy, shared resources and knowledge, and especially socialization tools like game chat features. I will also break down how some major free roam, "sandbox" games like Minecraft have provided their servers with the potential for developing extensive political systems. These systems provide server owners and moderators opportunities to structure and limit the behaviors of their player base and hopefully facilitate a positive playing environment.

The final section of analysis for this chapter will look at the extensive competitive, skillbased hierarchal system established in almost every major eSports title available. These systems, most often some modification of the Elo system, rank players based on their performance in- 
game and provide other players with an indication on an individual's relative skill. This helps the game's matchmaking mechanics place you within your designated skill bracket and keep the competition as fair and balanced as possible. However, this is not the only feature of the skillbased hierarchy in these games, as social prestige and status also play a huge role in how rank is perceived. To that end, I will also look at how more serious, competitive players compare to the larger, casual player base in these games and how their tastes and dispositions regarding the game influence the structure. Additionally, I will break down how some of these systems have changed and shifted over time to become more accurate and balanced when trying to rank players. Lastly, a look into how major video game streamers and professional eSports players influence the social hierarchy and the norms and behaviors of players in-game. By the end of this chapter, I will have established the interconnected relationship that player habitus and the structure of the video game world have on developing and reproducing a social hierarchy, as this interwoven relationship is key to understanding how forms of capital are produced in a variety of contexts.

\section{Literature Review}

\section{Social Hierarchy and Class Position}

To begin this review of the literature, an understanding of Bourdieu's conception of class must be established. In the general perception of class hierarchy, most individuals think of it in an economic context. In other words, we assume your position within the social structure is almost entirely determined by one's income bracket and access to resources. Bourdieu argues that this is a very limited way of understanding social positioning and states that, "a class or class faction is defined not only by its position in the relations of production... but also by a certain sex-ratio, a certain distribution in geographical space, and by a whole set of subsidiary 
characteristics which may function as real principles of selection or exclusion without ever being formally stated," (Bourdieu, Distinctions, p. 102). He is asserting that an individual, or a collection of individuals, can influence the class structure of a social space based on their tastes and dispositions (i.e. habitus) and can adjust their own position through an application of them. This will be essential for my analysis section, as I will argue that the player community of most games are important stakeholders in how the formal structures, socialization settings, and ranking systems come to be.

Bourdieu goes on to point out that an individual's position, and their future trajectory, within the social structure is based on a balance between the structure itself, "collected capital within the setting," and the inclusion of their inherited capital from other social environments, (Bourdieu, Distinctions, p. 109-111). Inherited capital will be an essential point of discussion for chapter four but for now, it is understood as the norms, practices, skills, etc. that an individual learns through familial connections or other close bonds and that they then, in turn, utilize in social settings. A good example of inherited capital in the context of video games would be a new player, having already learned skills of cooperation and teamwork from other settings, automatically being better equipped for success in-game than other new players. Inherited capital is also largely dependent on the economic and educational status of the player prior to joining the virtual social field. What I mean by this, and something Bourdieu points out, is that having access to better education and more economic resources places the player automatically in a higher tier within the game. They are more well-versed in problem-solving skills, cooperation, and even have a much higher likelihood of having more advanced gaming gear like headsets, controllers, etc., which will increase their skill position. Bourdieu states that individuals with a higher inherited capital not only operate differently in the same social space as those without the 
inherited capital, but they are also directly opposed to the tastes and dispositions of the latter group, (Bourdieu, Distinctions, p. 114-115). Inherited capital and class position prior to playing also can have an influence on the types of games being played and the individual's play style. According to Gailey, middle class families tend to avoid military, violence-based games, instead opting for the fantasy-odyssey types which focus on cooperation, character development and problem solving. Working class families look more at sports and FPS games that contain competition and conflict features, (Gailey, p. 84-89). These features will be looked at more extensively in the following sections of analysis to help us better understand the development of the social hierarchy within these games. Additionally, the influence of this inherited capital will be essential for understanding how the habitus of these game communities develops, as the top $<1 \%$ of the player population (i.e. streamers and professional players) largely dictate the playerbased decision making for these games, with the casual player's concerns receiving very little attention.

The final portion of Bourdieu's discussion that is relevant to this chapter, and that will help alleviate the disproportionate representation of the player base in the culture and decision making, is the reproduction and reconversion strategies available to actors when trying to adjust their class position. Reproduction strategies, according to Bourdieu, are a set of practices, "whereby individuals or families tend, unconsciously or consciously, to maintain or increase their assets and consequently to maintain or improve their position in the class structure," (Bourdieu, Distinctions, p. 125). These strategies are largely dependent, as we mentioned above, on the current composition of an individual's capital and the tools available to them for reproduction. Therefore, an individual with little access to resources and tools will struggle to increase or even maintain their current position. In the context of video games, specifically when 
looking at competitive/multiplayer ranking systems, players with little video game experience, lack of access to a good headset or controller, etc. will directly hinder their ability to be successful in the game. However, the pure skill of the player can also be considered a form of inherited capital and is something that players are born with, not unlike another sport or challenging activity, which will help counter the negative consequences associated with a lack of resources and technology. Actors can also utilize what is known as reconversion strategies, which allow the individual to reconfigure the current system in a way that allow for easier production and usage of capital for players. When looking at video games, player-led campaigns to make structure or design changes are a great example of individuals using reconversion strategies to shift the social field in way that benefits them. In the analysis section, I will look at some specific examples of players using reconversion to make their gaming environment more enjoyable and accessible.

Before moving on to discuss the manifestations of social hierarchy in the virtual world of video games, I need to establish what the proper definition of hierarchy is, as well as the various forms it can appear in. According to Björn Toelstede's research, hierarchy can be defined as "any form of social differentiation and stratification as well as differences in authority over others (i.e. power asymmetries)," (Toelstede, p. 335). For Toelstede, it is these power asymmetries that provide the basis for class structure and position in society and create a scale of hierarchy that nearly every major institution falls under. The first form of hierarchal structure defined by Toelstede is Networks, which actually do not contain a formal hierarchy and coordinate public goods at the peer level pro-socially. Individuals hold one another accountable at the citizen level, never escalating to elected officials, making it most comparable to a communist system, (Toelstede, p. 338-339). In the contemporary context, organizations that 
utilize this structure would be things like the stock market or social media, which often suffer from an inability to maintain that level of self-management. The next step in this scale is a Coordination Hierarchy, which operates a "pro-social vertical structure based on cooperation, public good contribution, and vertical escalation of public goods coordination," (Toelstede, p. 339-340). These are most comparable to modern democratic systems and are considered the most effective as they provide a balance of the inherent human need for structure and while also providing opportunities for self-determination. These are also highly meritocratic in that individuals are rewarded for contributing to the public goods. The third step in this scale, and the last of which that will pertain to this analysis, is the Dominant Hierarchy, which involves antisocial hierarchal structures that are the result of power sharing. Toelstede states that they are, "systems of obedience and command," and are built top-down with the aim of securing the elite's position within the structure, (Toelstede, p. 342-343). These are most commonly authoritarian or hybrid regimes that focus on a highly centralized form of authority. These three systems will be a heavy point of emphasis in the first analysis section as I examine the hierarchal structures in MMORPGs. Many of these games like WoW, or sandbox games like Minecraft, have structures in place that can and do facilitate the production of pseudo-political systems within the community. In this thesis, hierarchy will be viewed in the context of sociological institutionalism, which argues that hierarchal structures were put in place because it was and still is viewed as a functional and effective way of coordinating human interaction. Individuals, according to Miura, align themselves within these structures as a way of specialization and formalization of their activities, (Miura, par. 3). Hierarchy, in this line of thinking, is based on the division of labor as well as the formalization of an individual's role, relationships, and behaviors which are then established as the rules for interaction within that social setting, (Miura, 
par. 3-5). This conception of hierarchy finds overlap with Bourdieu's conception of social interaction in that it is a mutually supportive relationship between individual behaviors and the structures built around them. In the following section, I will examine how video games have worked to develop hierarchal systems within them and how they have directly and indirectly influenced player development and interactions with others.

\section{Hierarchy in Video Games}

Before moving on to examine the social and civic structures in both MMORPGs and FPS games, I need to establish what the hierarchy looks like in these games and how it reinforces class position in its player base. To begin, let's first analyze how social status for players is established and maintained in-game. Social Status in the context of video games, according to the TEFCA research institute, is the "extent to which a player is admired, esteemed or approved by the other players of the game as well as by persons outside of the game, (TEFCA, par. 1). Social status in video games is almost always directly related to the player's performance ingame, meaning that the more successful they are, the more recognition and respect they receive. There are also opportunities for players to increase their social status in games that allow for creative control and self-determination, which most often happens in MMORPGs like WoW. TEFCA's research points to competition and performance differences as the primary indicator for status in FPS's like Overwatch and Call of Duty. In the case of MMORPGs, the displayed character level and the variety of rare and unique character gear are used as methods for highlighting performance and status, (TEFCA, par. 2-5). Social structures in video games have become an integral aspect to the success of the game itself with the exponential expansion of MMO features. Large scale multiplayer games are now the standard for popular video games and players have a variety of reasons for playing these games. According to Nick Yee's typology of 
MMOs, there are five major features that facilitate this online gaming environment. The first feature, relationships, focuses on measuring the player's desire of developing positive social relationships with other players and players who score high in this typology find themselves utilizing the social features far more than others. The second feature, immersion, is something I discussed at length in chapter 2 and measures a player's desire to become ingrained and immersed into the virtual world. The third typology, grief, focuses on a player's willingness to engage in toxic and abusive behavior in game, most often in the form of team killing or destroying another player's property in-game. Achievement is the next category, which looks at how motivated players are to become powerful and complete in-game objectives. The final typology, leadership, looks at the assertiveness of the player and how willing they are to cooperate and lead others through missions and competitive matches, (Stenson, p. 3-4). These five major typologies help us to better understand the motivations for play and also the play styles of individual players. One of the most interesting features of how these social structures are formed is that video games, unlike many other forms of social interaction, lack the geographical proximity component that is traditionally assumed to be a key factor in facilitating socialization. Stenson points out that social groups form both on the basis of proximity to one another and on their willingness to engage with others based on choice. As video games often lack a geographical proximity component, these social groups are almost entirely predicated on choice, meaning that the social bonds established are based on the resources and social aspects being provided in the group itself, (Stenson, p. 7-10). There must be real value for the player to want to join a social group, which means these connections require strong relationship between the two individuals rather than just in-person accessibility to one another. 
When we look closer at MMORPGs, we see that the primary method for creating social interaction is through guilds or factions within the game. Game developers like Blizzard not only support these guilds, they actively promote player membership and socialization within them. These guilds allow players to part of visibly defined group within the game, providing player to player interaction and cooperation in an effort to facilitate the success and enjoyment of their community. Blizzard also offers a variety of guild management tools for the leadership to maintain the structure and hierarchy, which include a guild tag, in-game communication channels, item borrowing, guild statistics and even punishment features like bans or suspensions, (Ruggles et. al., p. 118). These features highlight not only an encouragement of socialization but also the development of social and even political hierarchy within the game that has a positive influence on how the player interacts with others and how they view the social field they are currently in. As I mentioned in the earlier portion of this chapter, human beings crave structure and socialization and video games are no different. Without formal structure a game seems confusing and unenjoyable; having that direction and purpose in the game makes it worthwhile. To help further facilitate this game developers have a litany of techniques available to them, both in-game and outside of it, to help bolster the stability and cohesion of their community. Some of these in-game strategies include the support of player organizations like guilds, encouraging competition between players, consideration of both casual and hardcore players, and implementing trade systems and encouraging the virtual economy. In terms of outside tools, developers look to provide official forums for players to interact and share ideas, ensure that there is effective communication between players and the developers and involving the player base in design and development decisions, (Ruggles et. al., p. 117-122). These efforts by game 
developers highlight the importance of social interaction and a stable hierarchy in these games at every level, from the base level player all the way up to the head creators at design studios.

The guilds in WoW and other MMORPGs also offer opportunities for skilled players to mentor new members joining the group, teaching them strategies for quests or the best ways to earn in-game currency. As Jane McGonigal points out, quoting Christopher Bateman's research, "players seem to really enjoy training their friends and family to play games, with a whopping $53.4 \%$ saying it increases their enjoyment," (McGonigal, p. 87). In this line of thinking there are three primary jobs offered to players when they join a guild, which they are responsible for being an active participant in. Guilds operate in much of the same way as a large scale organization in the real world, individuals fall into the hierarchal structure where they are assigned a job, work to increase their position within that structure through collecting resources, completing tasks, and being a good support system for other members. The first job available to players is farming, which involves a repetitious task of exploring the world collecting resources like minerals and herbs that are then used to create items or potions that can help protect or buff characters when engaging in quests. The second job, crafting, involves taking the collected items from the players responsible for farming and turning them into weapons, potions, or character buffs for quests and raids. This job requires more specification, as certain professions in game can only make certain types of materials (i.e. herbalist can only make potions). The final job, mentoring, involves guiding new members of guild through teaching them new strategies or even providing loot to help get them started, (Rapp, p. 3). These jobs are essential for establishing the hierarchal structure within the game I will return to a discussion of them in the analysis section.

There are clear indicators of how MMORPGs like WoW facilitate the development of social and political hierarchies in-game, but what is less apparent is how competitive FPS games 
like Overwatch and Call of Duty do the same. When looking these games, we have to analyze them based on their competitive nature, meaning that the hierarchal structures within them are largely dictated by player performance and to some extent, their ability to socialize effectively. When looking at them in this context, failure is an often-overlooked positive aspect. Jane McGonigal highlights this feature, quoting the MIND Research Center's study for effect, "players exhibited the most potent combination of positive emotions when they made a mistake... excitement, joy and interest shot through the roof when they lost," (McGonigal, p. 66). Negative motivation is a key aspect of competitive FPS games and helps to be reinforce current class position but also encourage players to find new ways to increase that position. In other words, it facilitates the development of the reproduction and reconversion strategies that Bourdieu points to. Positive reinforcement for players is also extremely important in this environment, as a dejected player who consistently losing is unlikely to return to a game. This is why so many competitive FPS games work extensively to provide positive reinforcement, punishment for toxic behaviors and effective/accurate placement systems. First and foremost, video games like Overwatch, Call of Duty and League of Legends struggle severely with a toxic culture in their player bases and often lack the ability to address it. One of the major features implemented by Riot Games, the developers of LoL, is the Tribunal System which I discussed in Chapter 1 . This system provides a shaming mechanism for players who were reported to have been engaging in toxic or abusive behavior. If the report is warranted, players will receive a suspension or ban for a set period of time and are publicly advertised as bad player on LoL's main website. They do offer an appeal system, through which players can have their bans removed if they prove the report was incorrect or if they show remorse for their actions and work to avoid those types of behaviors. This system has proven to be an effective method for reducing 
toxicity in game, as it has seen a noticeable decrease in abusive chat logs or griefing in-game, (Soderberg-Rivkin, par.6-8).

On the flipside, games like Overwatch and Call of Duty offer positive commendations for being a good and fair teammate. In Call of Duty's social score system from their title, Call of Duty: WWII, players work to increase their social score by commending other players through in-game prompts, completing in-game challenges, and increasing your account rank through a prestige system (this is something I will discuss fully in the analysis section). Ranking up one's social score provides players with unique items like gun skins or in-game currency as levels increase, (Call of Duty Staff, par. 4-10). In Overwatch's case, they offer an endorsement system which provides three in-game options for promoting a teammates' performance. These are sportsmanship, which is associated with being a good teammate or a fair opponent. A very effective informal way of positively interacting with the lobby in this way is saying GG (good game) in the chat logs. This is a universal sign of sportsmanship for video game players, making it an extremely effective tool for positive socialization. Another endorsement available for Overwatch players is the Good Teammate option, which lets your teammates know that they have proven to be helpful throughout the game (i.e. healing/reviving teammates) or simply engaged in effective communication. The final endorsement, Shotcaller, promotes players who displayed effective leadership qualities that led to the success of the team. This final option both positively impacts the socialization as well as established a temporary hierarchy within the lobby, as the player receiving the Shotcaller endorsement is likely to continue to be the leader regardless of team dynamic, (Overwatch Wiki, Endorsements). An understanding of the process that both MMORPG and FPS developers have when facilitating social and political interaction within their games. Moving forward I will look more intensely at how features like guilds in the 
case of MMORPGs and competitive ranking systems for FPS games are not only creating social and political interaction but also how they have external benefits for individuals in other social environments as well. At the conclusion of this chapter a clear picture of the social and political hierarchies in these games will come into focus and we can better address how to promote and facilitate positive outcomes from them.

\section{Social and Civic Hierarchy in the Virtual Field}

\section{Building Social Structures in MMORPGs}

Now that the I have reviewed all prevailing literature surrounding the subject matter of this chapter, let's move forward and look at the varying structural and behavioral dynamics of guilds in WoW. When looking at motivations for playing MMORPGs, Zhang and Kaufman point to three major motivators, which are achievement, sociality and immersion. Since I have already discussed the research provided by these authors in chapter 2 , specifically the aspects of immersion, I will just briefly touch on the first two again. In terms of achievement, they find that players are most concerned with advancement of their character's abilities and power level, the underlying mechanics of the game in order to optimize performance, and competition against other players, (Zhang \& Kaufman, p. 152-153). In the context of WoW and its guilds, we can see that many of them facilitate competition between members and against other guilds, provide extensive opportunities for advancing and strengthening one's in-game character, and great mentoring opportunities to help teach players tricks and strategies that can work around some of the tougher mechanical challenges of the game. The second motivator, sociality, is primarily concerned with socializing relationships and teamwork. Socializing relationships refers to having interest in helping and supporting other players and developing long term relationships with them, (Zhang \& Kaufman, p. 152-153). Guilds offer a plethora of opportunities to both organize 
and cooperate as a team as well as develop meaningful relationships with your fellow guild members. These social relationships can and do extend beyond them game as well. Zhang and Kaufman point out that of the friendships made through the medium of WoW, over $75 \%$ of them become positive social relationships in the real world. Additionally, they noted that, in terms of guild organization, leadership and other guild members, $70 \%$ of players reported satisfaction, (Zhang \& Kaufman, p. 156-161). This is a fascinating statistic, especially considering how low approval ratings normally are for leadership in a social or political context. Players find themselves enjoying and appreciating the supportive environment facilitated by guild membership.

Now it must be asked; what does this approval rating mean for the cohesion of the community as a whole? Poor and Skoric, through their extensive research into MMORPG communities, find that guilds in WoW allow players to establish a long-term sense of social connection of achievement by leveling up the guild and adding to its resources, (Poor \& Skoric, p. 2266-2267). Players find deep pride in being successful in these guilds in a very similar way to real-life organizations and, in turn, feel responsible for their actions while being a member. For this reason, players tend to stay faithful to their current guild, as it rewards those who stay and help grow the membership pool. Poor and Skoric point out that around 65\% of all WoW characters maintain their current guild membership over a 3-year period, (Poor \& Skoric, p. 2269-2271). This data provided by the authors highlights how these games facilitate not only cooperation and teamwork, but also maintaining group identity and builds community networks that lead to civically engaged players within the servers. What's is most interesting about these bonds being made, and what makes them different from real world relationships, is the distortion of time and space within these virtual worlds. Lau points out that these relationships, due to the 
speed and intensity at which individuals engage in game-based activities, form and stabilize in a matter of hours and days, far faster than an in the real world, (Lau, p. 379-380). Human beings understand the passage of time based on the interpersonal connections we make with others, which is becomes muddled by the non-linear and dynamic way in which we interact with other players in-game. Time can be stopped and started within the game, meaning the player can remove themselves from the world only to return to the same point they left off. It allows for flexibility and freedom to engage with others and make meaningful relationships with them. Guilds do, however, vary pretty significantly in terms of their primary objectives and, in some ways, their hierarchal structure, which I will address next.

Dmitri Williams has studied MMORPGs thoroughly and has found great supportive evidence of stable social and political hierarchies within the world of Azeroth. Williams points out that of the many guild available, nearly all fall under one of four categories. The first category, Social Guild, has its primary goal as socialization between guild members. These social bonds are often extensions of real-world friendships that spill over into the game. PvP (player vs. player) Guilds are the second option, with its primary focus being battling against other guilds, most often in the battlegrounds or arena. Raiding Guilds find their efforts in-game being directed at organizing and scheduling 40-member team events than can last anywhere from 2 to 8 hours. These guilds require extensive coordination and time management on the part of the leadership, requiring a more stabilized and intricate political hierarchy within. The final option, Role-Play Guilds, work on a meta level in which players adhere entirely to their character's "role" within the guild and the world in general. This guild places heavy emphasis on the jobs available to guild members, (Williams, p. 10-13). Looking back at the discussion of the three primary jobs available to players (i.e. farming, crafting, mentoring), Role-Play Guilds take these 
extremely seriously, requiring players to make regular contributions, trade and interact with other guild members, all while staying in-character. This diversity of hierarchal structure in these guilds is very informative in regard to the complexity of social interaction within the WoW servers. Players not only seek structure and stability but also a set of objectives, access to resources, and positive social connections. To further strengthen the hierarchy within these guilds, many of the leadership utilize formal practices like mission statements, recruitment tactics and even regular elections for leadership positions in an effort to make members feel responsible and engaged with the concerns of the guild as a whole. Williams points out that guilds with clear policies and procedures are far better at managing tasks and had overall happier members, (Williams, p. 13-18). This again speaks to the point I made earlier that humans crave structure and social interaction, regardless of the medium. Speaking of leadership, Williams points out that most mid- to largescale guilds have a Guild Master in charge of macrolevel decision making and delegation to the various other leadership positions within the guild. The structures of WoW guilds, while on the surface seem autocratic and centralized, do indeed provide democratic methods of interactions to its players. As I mentioned above, many of these organizations hold regular elections and public forums to include all members in the decisionmaking process while still have stable leadership hierarchy to maintain the goals of the guild as a whole.

These guilds, thanks to the formalized social interactions through the hierarchal structure engrained within them, provide players with moments of democratic interaction that further stabilize their actions in-game as meaningful and influential. Returning to Lau's discussion, he points out that discourse between players becomes an extended moment of semiosis and an example of semiotic democracy, (Lau, p. 383). What he means by this is that the regular 
participation within this virtual world and engaging with other players is a way to constantly negotiate the framework and validity of the world itself. It is this interaction between players that makes the virtual world of WoW meaningful and creates explicit value for the community. Blizzard has clearly created a space within the WoW universe that is both socially interactive as well as a medium for learning and educating others about effective social and civic discourse. Next, I will analyze how Minecraft and its free roam, sandbox design has created opportunities for players to create and moderate extensive political systems within their server.

In Chapter 1 I analyzed Rolfes and Passig's research regarding proto governance in Minecraft servers, which I would like to incorporate into this discussion as well. Previously, I broke down the two most likely forms of government within Minecraft servers, constitutional monarchy and benevolent dictatorship. The former places the server owner and a few select moderators at the top of hierarchy that are responsible for nearly all major decisions within the server. They still, however, are responsible for listening and adjusting to the needs of the player base and must use tools (i.e. plugins) to make sure server members are playing fair and are avoiding griefing other players, (Rolfes \& Passig, p. 8-11). This structure operates in much of the same way as guild hierarchy does in that the leadership is very centralized but still required to adhere to the low-level members and their concerns. The latter of the two has the same, top down leadership strategy but differs in that it ignores the wishes of the player base, with every decisions and exercise of power coming from the server owner and at times a small number of moderators. These servers very rarely have plugins in place that limit or punish toxic behaviors, which leads to many players becoming frustrated and look for new servers to become a member of, (Rolfes \& Passig, p. 6-8). These two server designs are primarily used to allow for more freedom for server owners and moderators to build their server to their liking. Most individuals 
who own or moderate a server want to enjoy not only high levels of autonomy in the structure of the server, but also the microlevel activities like farming or mining resources. Allowing for selfdetermination and having a lack of checks and balances on the player base within these servers removes a good deal of responsibility from moderators in regard to toxic and abusive behavior. This again, provides them with a substantial amount of free time in the server to focus on personal goals that would otherwise be spent mediating player disputes or removing individuals from the server. With this in mind, there are two other major examples of political Minecraft servers that must be analyzed to see the full scope of political ideologies within this setting. These two servers are the Civcraft, which is a democracy-based environment with strict rules and limitations that players must follow in order to maintain their membership, and 2B2T, Minecraft's oldest anarchy server which has run for over ten years and lacks any sort of formal structure or organization.

Let's first discuss Civcraft, as it exemplifies the intensification of formal hierarchy within sandbox games and how it can be used to facilitate democratic discourse between players. According to their official Reddit page, Civcraft is unlike most other Minecraft political servers in that it uses the Citadel protection plug-in, which allows individuals to defend their buildings and resources from griefng by increasing the amount of damage certain building materials can take before being destroyed. The increase in protections for clean stone, iron and diamond is significant enough that it largely deters toxic players from engaging in destructive behavior. As an additional protection, players are given "precious stones" which denote an 11-block radius, in which the player who owns the space of land is notified that someone has entered and is attempting to either break or steal items (Civcraft, Reddit). The precious stone plug-in acts like security camera for players to ensure that while they are away from their base, they can still 
protect it or at least seek punishment against the individual who griefed them. In terms of democratic aspects, Civcraft has another plugin called RealisticBiomes, which makes farming harder by limiting growth rates of crops and making them only grow well in certain biomes," (Civcraft, Reddit). In conjunction with the RealisticBiomes plugin, Civcraft also provides a FactoryMod, which allows individuals to produce raw materials through a factory system but for a higher cost of in-game currency, (Civcraft, Reddit). This scarcity therefore necessitates civic engagement and democratic discourse between players to not only survive, but also to help the community thrive as a whole. To further facilitate supportive interaction between server members, the Civcraft server also offers a trading shop, in which players can directly trade resources with one another for precious gems like gold or diamond, (Civcraft, Reddit). This creates a stable in-game economy all on the base of the players agreeing to the rules of the server, as it is not a design feature of Minecraft like it is in WoW. In addition to these economic and social interactions, Civcraft also contains a variety of public forums, both within the virtual world and on the server's official Reddit page, for players to engage in civic discourse regarding a litany of topics related to accepted player behaviors, prices of goods, and access/allocation of resources overall, (Civcraft, Reddit). All of these features speak to the efficacy of the democratic institutions within the server, as this would be impossible without a formal hierarchal structure.

The final point of analysis in this section, Minecraft's 2B2T anarchy server, is one of the longest standing Minecraft servers in existence, with an active time of over 10 years. In standard Minecraft, the player is dropped in a spawn location in a randomly generated world and must fight monsters and collect resources in order to survive. The 2B2T server takes this mindset and allows it to run free with hundreds of server members having complete free will and total lack of consequences for their actions. Kiberd points out that players in this server are advised to hide 
their loot underground and be prepared to be killed by other server members constantly. The first era of 2B2T server began with players expanding their map view and establishing bases around the world. Players began to align with specific factions within the server as a way to protect themselves from other players. The server members began to operate like largescale gangs, with players joining together for raids of other groups' compounds and actively attempting to kill new members at the spawn point, (Kiberd, par. 6-10). What really makes this server anarchic is the lack of moderation by the server owner through tools like plug-ins or player bans. The intention of the server is to operate like a free-for-all, everyone for themselves. Hausemaster, the founder of $2 \mathrm{~B} 2 \mathrm{~T}$, states that his intention for creating the server was that, "I wanted to see what destruction could be made, but also whether there would be connections between players in such a chaotic rule-free environment," (Kiberd, par. 25). I would say that both of these objectives set by the server owner have been achieved, as it has become one of the most violent Minecraft servers active. Additionally, it has provided extensive insight to the point I made previously that human beings crave structure and interaction. Even in the most chaotic and disorganized formation of a Minecraft server, players still find themselves drifting towards being a part of group, something bigger than themselves. Sometimes this membership is out of necessity, as it likely is in this case considering how dangerous it is to traverse $2 \mathrm{~B} 2 \mathrm{~T}$ alone, but it most often spawns out of our need to make connections with others. Now that I have fully fleshed out the social and civic nature of the structure of MMORPGs and sandbox games, I can shift focus towards the competitive hierarchal structure of FPS games like Overwatch and Call of Duty. 


\section{Competitive Ranking Systems’ Hierarchal Structure}

MMORPGs have clearly proven themselves to be effective environments for producing social and civic hierarchy, so now I can move forward to look at the skill-based hierarchal systems that form in FPS games, as well as how they impact the social connections being established. When looking at these connections, players must make decisions on who they want to play with, ranging from friends and family to playing with strangers or joining a clan (i.e. social group like a guild). McQuarters' research focuses on these social relationships between players and notes that all respondents from his study reported playing with real life friends or immediate family. In this setting, players are deriving enjoyment from the shared sense of achievement, which further bonds them together. Additionally, he points out every respondent also reported playing with at least one stranger but did state that many of them reported dealing with toxic or abusive behavior from the random player. Finally, McQuarters found that of the 12 people interviewed, only 6 reported being a part of a clan in a major FPS title but 10 of those 12 said they were a member of a guild in an MMORPG. Some reasons for joining these clans were playing competitively, learning new tactics for the game, always have team members to play with, and helping to mentor new players, (McQuarters, p. 35-45). One of the reasons for lower membership in clans for FPS games than their counterpart in MMORPGs is that there is more inherent value in being in a guild, as it provides resources, training and more direct socialization that does not always come with being in a clan in FPS games. The hierarchal system in games like Overwatch and Call of Duty struggle from structural barriers to newcomers that limit their ability to be a part of these social interactions. One of the most influential of these barriers is game communication, or "callouts". Callouts are phrases and nicknames given to map locations, strategies, and team dynamics and are often complex and unique to each game, making the 
entrance into the social field challenging. Once a player has moved past this barrier however, callouts are proven to be one of the most effective ways of establishing social bonds and facilitating proper team hierarchy, (McQuarters, p. 45-48). The unique lingo of the game makes the player feel a part of larger culture and social field that they can find a good position for themselves in.

The most prominent barriers to social interaction for these games, however, are actually the ranking systems themselves. Most FPS games have two, semi-related ranking systems within them, a causal, public match system and a competitive Elo-based system. For this discussion I will be looking at how Call of Duty's public match "prestige" system has shifted its design over time to be more accommodating to new players and how it differs from the competitive ranked play system. Prestige mode has been a staple of Call of Duty since the early days of development and is their way of displaying a player's skill level and time invested, culminating into their overall status within the game. Beginning with Call of Duty 4: Modern Warfare, the prestige system has changed dramatically over time, incorporating a variety of new features. For instance in Call of Duty 4, players need to reach level 55, at which time they could enter prestige mode which granted the player a new icon next to their name as well as new challenges but it would remove all earned guns, gun skins, and any other unlocked in-game item, (Call of Duty Wiki, Prestige Mode). This was intended to make players really consider increasing their prestige status and also gave them an opportunity to start fresh and begin working on the challenges and gun skins again. This almost operated like a reset for class position, as it did not reset their skills, but it reset their access to the best guns and attachments in-game, making it more challenging to succeed. Jumping forward a few titles to Call of Duty: Black Ops, we see this prestige system change dramatically, with all new leveling and unlock 
systems. In Black Ops, players earn in-game currency called CoD points which can be used to unlock guns and attachments as opposed to leveling up to gain access to them like in older titles. Players are also able to gain additional custom class slots (increases from 5 all the way up to 10 slots after each prestige), keep all emblems, backgrounds and game modes that were unlocked during the level up process, (Call of Duty Wiki, Prestige Mode). CoD points are reset upon prestige as well as weapon unlocks, but the process for getting them back is far less challenging than previous ones. This new system allowed players to pick and choose the guns and attachments they wanted to unlock, making it easier for them to get in a good skill position within the hierarchy without having to wait 20, 30, 40 levels to use the items they enjoy. Another major advancement that the Call of Duty franchise made to their prestige system was creating prestige ranks for their PvE experience, Call of Duty Zombies. In the Zombies game mode, players fight hordes of zombies in a round-based system in which the number and strength of the zombies increase with each round, (Call of Duty Wiki, Prestige Mode). Adding the prestige system into Zombies not only provides non-PvP players with opportunities to gain prestige and status within the game, but also for earning valuable rewards for leveling up. The prestige system in Call of Duty has clearly gone through some drastic changes since its creation and these changes have worked to facilitate a positive hierarchal structure that rewards players for time played and does not punish players for performing poorly. These systems are built for the causal players at the lower end of the hierarchy and help to keep them playing and avoid burnout. These systems do not do enough for the high-skill competitive player, however, as it does not challenge them or highlight their true performance based on wins and losses. That's where the Elo-based ranked play systems come in. 
In both Chapters 1 and 2, I discussed Elo based ranking systems and their ability to facilitate the production of teamwork and problem-solving skills. In this chapter, their value comes from their ability to create a hierarchal structure based entirely on the skill and performance of the player, pushing them to work om learning new skills and increasing their current position. As I pointed out in the previous chapter, nearly all modern ranked systems use the TrueSkill system, as it provides the most accurate placement for players based on their wins, losses, and draws, with the performance and ranks of both teammates and opponents when making its calculation. In Call of Duty's system, individuals are placed in ranked tiers, ranging from Iron all the way to Master division, similar to Overwatch's ranked play that I mentioned in Chapter 2. In addition to using this placement system to establish a hierarchy, the game's developers also ban certain guns, attachments, character buffs and killstreaks (large, mostly aerial automated attacks like a helicopter that a player earn after reaching a certain streak of kills), (Call of Duty Wiki, League Play). The bans implemented are intended to provide a fairer competitive environment and avoid the unbalanced and unpredictable nature of certain weapons and killstreaks. Most of these bans in recent years have been decided on by surveying the professional eSports players to determine what they find to be the most imbalanced aspects of the game. This system speaks to the importance of the hierarchal structure even in establishing itself. As Bourdieu pointed out, those at the top of a specific tier within a class structure largely dictate the decision making and social conditions for that group and video games are no different. The pro players opinions and advice are valued at a much higher rate than they average player, as they have more experience and skill that help them determine the pros and cons of a game title. A much clearer picture of the skill-based hierarchy systems of FPS games has now been established, completing my analysis section of this chapter. The class structure has proven 
to be effective at placing individuals into their correct position within the hierarchy and providing levels of status and prestige to their players in order to make their efforts have meaning and value to them and to other individuals within the game.

\section{Conclusion}

In the chapter, I have established and provided evidence for my argument that video games can and do operate like social and political hierarchies, through which players learn valuable social and civic skills. These structures also provide numerous opportunities for developing social connections with other individuals in the same setting. This chapter began with a reexamination of Bourdieu's theory of practice with a more direct look at how class position and social hierarchy are established, with supportive evidence of what traditional examples of hierarchal systems look like. Following this, the remainder of the literature review was spent establishing what the social and civic networks within video games look like, as well as the structure and design features of both MMORPGs and FPS games facilitate this in their respective fields. This section provided us with an extensive theoretical framework for how to analyze these social structures and determine how individuals find their position within them.

With that in mind, I moved on to the analysis section, which was broken into two parts. The first part, which focused on the social and civic structures within MMORPGs, helped highlight the importance of guilds and other social groups within these virtual fields. These systems provide players with the structure they crave, access to shared resources, teammates available for cooperative missions, and even mentoring opportunities for players to learn about new tactics and skills. As I noted earlier in the chapter, guild leadership and decision making has around a $70 \%$ approval rating from members of the guild, making it far higher than most political and corporate organizations. I have provided evidence that this is due to the more direct 
influence that entry level members can have on the decision making, with elections and public forums being an essential aspect of the development of these guilds. Guilds, therefore, operate in much of the same way as real life social and political organizations. However, they become noticeably more democratic when they provide an avenue for direct influence in the decisionmaking process for their members, even with a centralized form of authority. Following this analysis, I broke down the varying proto-governance Minecraft servers ranging from benevolent dictatorship, to attempts at democracy, to the 2B2T anarchy server. Each of these had their own unique features that made them interesting and good examples of hierarchal structures, as well as provide my analysis with evidence that formal hierarchal structures can be formed entirely within the context of a virtual world.

In the second analysis section, I examined the skill-based hierarchal systems that are so prevalent within FPS games like Call of Duty. I pointed first to the causal, public match prestige systems that allows players to level up their social status through playing games and completing challenges. This system allows causal players to both be a part of some form of skillbased hierarchy as well as earn visible prestige with others in the game. In addition to the prestige system, I also analyzed the development the competitive ranked play in Call of Duty, which sets unique rules and bans for guns and attachments in an effort to make a more balanced gaming environment as well as make it more competitive. Both of these systems proved to be effective at creating and stabilizing a hierarchal system based on meritocratic terms, something many major corporations and other organizations attempt to do every day. Overall, this chapter has highlighted the efficiency of game developers at creating stable structures for ordering and classifying video game players based on their performance and time spent playing. Now that I have confirmed my assertions at the beginning of this chapter, I can now move forward to the 
last portion of my research. The fourth and final chapter of this thesis will look at how the relationship between the two produces varying forms of capital (i.e. social, cultural, material, and symbolic) and how they can be converted and utilized in a plethora of contexts. 


\section{CHAPTER IV: PRODUCTION AND UTILIZATION OF CAPITAL IN ONLINE GAMING}

\section{Introduction}

Using Pierre Bourdieu's theory of practice, I have now established the variety of norms and values within the video game community, as well as the impact the hierarchal system and the virtual social field can have on the production of those norms and values. There is one key aspect missing from this analysis that will be the primary focus of this final chapter. Bourdieu's theory places a heavy emphasis on the production and conversion of three primary forms of capital: economic, social and cultural. Additionally, he expounds upon the concept of symbolic capital, which is a representation of an individual's prestige and status within society and manifests as a combination of the other three forms. This chapter will examine how these four forms of capital are produced, how the virtual world of video games facilitates that production, and how these can be applied outside of the context of online gaming.

The first section provides an in-depth analysis of Bourdieu's definitions of capital, with supportive research regarding this topic from other notable authors in the field. This investigation into the foundational aspects of capital will provide my research with a solid footing when trying to pinpoint the same aspects within the context of video games. It will additionally highlight the importance of these forms of capital when engaging in social, political and economic interaction.

Following this breakdown, I will then use the remaining section of the literature review to discuss what cultural, economic, and social capital look like in the context of video game play, as well as how they culminate into what many contemporary authors have coined as "gaming capital." Gaming capital, for the purposes of this thesis, will be understood as a "way to discuss the role that knowledge, experience, and skill have both for an individual, but also for the larger cultural and economic system that surrounds digital games," (Märyä, p. 6). In other words, 
gaming capital is a form of symbolic capital in the setting of video games that has a direct influence on the player's experience when playing and interacting with others and can also positively impact the individual outside of the context of the virtual field.

The analysis section of this chapter will be broken into four parts, each focused on a specific form of capital and how it manifests within video games. In the first section I will look at the culture of video games and how it produces its own unique brand of cultural capital that is both contained within the virtual setting but also has enormous impact on the larger cultural field in modern society. Following this, I will analyze the varying ways in which players can increase their economic capital through game play, as well as how the inherited capital individuals possess can positively impact their status within the game itself.

In the third section of analysis, I will look again at the social features of video games, something I have discussed at length in the previous three chapters, and how they influence the production of social capital. This section will analyze the importance of social connectedness when playing video games, as well as the social and civic value of them and how social capital acquisition varies based on the type and style of game being played. The social relationships established, and the capital produced through them, also have noticeable influences on our reallife relationships and interactions so I must also include a discussion of this conversion. The final portion will look at the production of gaming capital, what it can actually be defined as and how it manifests in-game. I will reassert the importance of status and prestige within the video game community, something I examined extensively in chapter 3 , which will require a return to the discussion of ranking systems and guild/clan structure in video games. By the end of this chapter, I will have fully fleshed out the varying forms of capital and how they manifest and influence the world of video games. 


\section{Literature Review}

\section{Bourdieu's Forms of Capital}

To begin the review of the literature surrounding the concept of capital, I must first establish what Pierre Bourdieu's definitions of each are, as well as providing additional support from other author regarding these forms. As a starting point, I need to look at economic capital, as it is the most basic form of capital and easiest to conceptualize among them. Economic capital refers to what we traditionally think capital as; in other words, the development of monetary value through the production and use of goods or services. Additionally, as Bourdieu points out in Forms of Capital, "economic capital is at the root of all the other types of capital and that these transformed, disguised forms of economic capital, never entirely reducible to that definition, produce their most specific effects only to the extent that they conceal (not least from their possessors) the fact that economic capital is at their root, in other words - but only in the last analysis - at the root of their effects," (Bourdieu, Forms of Capital, p. 11). This is an essential aspect to his argument, as it highlights the inherent need for humans to be able to convert their skills, values, etc. into something that can increase their economic status within society. Economic capital dictates how we produce and utilize the other forms of capital in society, framing everything we do in an economic context. Bourdieu argues however, that this is an inaccurate way of looking at how we interact with one another and we need to place a higher value on the other forms of capital and their unique impact.

Moving forward, when looking at the concept of cultural capital, Bourdieu points out that there are two primary areas of production for culture. He argues that "legitimate culture," is facilitated simultaneously by the academic setting in which the individual is in, as well as their inherited cultural capital from familial ties. Legitimate culture refers to "scholastically 
recognized knowledge and practices, manipulated by the dominant class," (Bourdieu, Distinction, p. 23). An individual's position within the social structure is largely dependent on the access to legitimate culture and the use of it, meaning that those who lack the inherited capital or high-quality education have severely limited mobility within the hierarchy. One of the most notable ways for individuals to shift their position without access to legitimate culture is through the use of illegitimate or extra-curricular culture. Bourdieu states that this refers to the, "production and accumulation of knowledge and practices outside of the control of educational institutions," (Bourdieu, Distinctions, p. 25). These include self-taught skills, experiences and knowledge learned through everyday practices and behaviors. Good examples of producing illegitimate culture would be through social interaction on a sports team, in a club or even just out to dinner with friends. In these settings, individuals produce cultural capital through interacting and intaking the cultural perspectives of the other people within that social environment.

Continuing the discussion of cultural capital, Bourdieu posits that there are three primary forms in which it manifests. The first of these is the objectified state of cultural capital, which refers to cultural goods like art, music or books. He states that, "Cultural capital in its objectified state presents itself with all the appearances of an autonomous, coherent universe which, although the product of historical action, has its own laws, transcending individual wills, and which, as the example of language well illustrates, therefore remains irreducible to that which each agent, or even the aggregate of the agents, can appropriate (i.e., to the cultural capital embodied in each agent or even in the aggregate of the agents). However, it should not be forgotten that it exists as symbolically and materially active, effective capital only insofar as it is appropriated by agents and implemented and invested as a weapon and a stake in the struggles 
which go on in the fields of cultural production (the artistic field, the scientific field, etc.) and, beyond them, in the field of the social classes," (Bourdieu, Forms of Capital, p. 6). What Bourdieu is explaining here is that these physical objects are representations of cultural capital, insofar that it is taken and utilized by agents of culture and can only have true value as a form of capital if those individuals who are invested in this field apply that value. When we look at video games more intently, we will see how both the developers and the game community play a role in establishing the value of this form of capital. The second form of cultural capital, the embodied state, refers to the language, mannerisms and preferences of individuals in a social setting. These are a combination of the inherited cultural aspects we get from friends and family, as well as the acquisition of new forms of culture as well. This is culture manifesting through the way we talk, act, and think within a social setting. In other words, this helps to explain the production of the habitus within a social field and, in the context of video games, helps explain how the widespread gamer culture has come to be.

The final form of cultural capital is its institutionalized state, something that is essential for understanding the development and stabilization of the video game community as a whole. Bourdieu states that the institutionalized form of cultural capital refers to the, "objectification of cultural capital in the form of academic qualifications institutionalizes the recognition of the cultural capital possessed by any given agent and also makes it possible to compare qualification holders and even exchange them," (Bourdieu, Forms of Capital, p. 7). This speaks to the importance of "legitimate culture" through the education systems, as this form helps us to establish an individual's position within the social hierarchy and also make the value of cultural capital convertible into an economic context. As I said above, this helps to explain why creating a stable culture surrounding a social setting is so important and why the video game community 
has to work not only to promote a culture, but more specifically, the right kind of culture. What I mean by this is a culture that promotes diverse viewpoints, positive socialization and opportunities for civic engagement. Developers have to work to promote positive values and dispositions between players, which as I mentioned in chapter 1, is a daunting task to say the least.

Now that the importance of cultural capital has been established, let's discuss the development and impact of social capital. Bourdieu states that social capital is, "Aggregate of the actual or potential resources which are linked to possession of a durable network of more or less institutionalized relationships of mutual acquaintance and recognition, which provides each member w/ backing of the collectively owned capital," (Bourdieu, Forms of Capital, p. 8). Social capital production for an individual is largely dependent on the size of their social network and how effectively one can mobilize those connections in tandem with their own stores of capital. Social capital is also extremely influential on our overall class position and we create movement within that space. Vertical movement within a hierarchal system refers to moving up or down within the same field (i.e. individual receiving a promotion in their field of expertise) and primarily dictated by their accumulated cultural and economic capital. Social capital, however, has a huge influence on our transverse movement, which refers to moving between two, seemingly unrelated fields. Social capital helps facilitate new relationships in fields we are previously unaware of or have no reference to. Utilizing capital that an individual can produce in these social settings, they facilitate the development of new relationships in emerging fields and open doors that may not have been available previously. Social capital, along with cultural capital, also influences how we interact with one another. Through socialization, we learn the acceptable and unacceptable forms of interaction in these settings, and, in the process, 
unconsciously adjust our usage of social capital. When we build relationships with one another, the interaction teaches us about varying political and cultural values of others, shifting our own perspectives when entering into new social fields.

The final form of capital in Bourdieu's theory is symbolic capital, which refers to the “intangible, culturally significant attributes like prestige, status and authority," (Mahar et. al., p. 13). Prestige and status within a social field is something I examined to great extent in chapter 3 where I discussed the influence of both the virtual setting of video games and the norms and values of the community at large in the production of these features. The production of symbolic capital is not easily defined, as it requires an accumulation and overlay of the other forms a capital to manifest and utilize it in any social field. As Ihlen points out, quoting Bourdieu's work, all three types of capital can take the form of symbolic capital when they are "grasped through categories of perception that recognize its specific logic or, if you prefer, misrecognize the arbitrariness of its possession and accumulation," (Ihlen, p. 5). What this means is for the other forms of capital to manifest as symbolic capital, the individuals in that social field must attribute value to them in the sense that they can impact the status and social position of that individual. For example, someone may have access to extensive cultural knowledge about a niche subject but if it does not have inherent value in the specific setting, it will have little to no impact on their movement within the hierarchy. In the context of video games, symbolic capital is best represented as gaming capital, as it is a combination of the other three forms of capital and represents a player's status within the game world and the community overall. As Ihlen posits, actors within a social field are distributed within that field based on two primary factors; the overall volume of the capital they possess and the composition of that capital (i.e. how much of each form they possess), (Ihlen, p. 4). For player to able to utilize gaming capital properly, they 
must find an effective balance between their own inherited social, economic and cultural capital, as well as incorporating newly discovered manifestations of each. In the next section, I will look at how video games have worked to not only provide areas of production for the other three forms, but also how these can be used to increase their gaming capital and positively impact the player's experience.

\section{Forms of Capital in Video Games}

Since economic capital is easily understood and its impact on video games is related more so to actual examples of it in-game, I will save the discussion of it for the last section. Instead, I will briefly discuss how video games are actively facilitating the production of social and cultural capital, as well as how they culminate into gaming capital for the player. To begin, I am going to look at the development of video game culture through the lens of Bourdieu's conception of cultural production. As video games have advanced, becoming more complex social environments, the culture of video games has advanced with it. From the perspective of the casual gamer, serious, "hardcore" gamers are viewed as anti-social nerds lacking proper social relationships. Nichols argues that this is in fact not true and states that video games produce their own subculture, which is largely dictated by the community of players not the developers. This culture is facilitated both through interaction in-game (i.e. learning the language, strategies, and dispositions of other players in the virtual setting) and through third party services like social media and forums. The weight and importance of the actions in-game are determined by these players through a variety of tools available to them. As Nichols states, "players may come together and craft their own meanings, and in fact be encouraged to do so by

the industry or other groups. Certain forms of modding or cheating are examples of this. Creating new content via mods or taking advantage of sanctioned forms cheating such as Easter Eggs and 
communicating with other players about them would be seen as expressing, and potentially generating, new cultural and social capital by creating or extending the cultures or subcultures around a particular game,” (Nichols, p. 43). Cheating and in-game modifications are great examples of the expansion of the gaming culture, even if it is outside of the bounds of the accepted rules of the game.

Video games also have an inherent impact on our understanding and absorption of exterior cultures and ideas. In their research, Shliakhovchuk and García find a number of positive impacts on our understanding of content, how we acquire knowledge and how we perceive certain social and cultural factors in our lives. For example, the authors found that when exposed to video games, players have a higher understanding of the lack of racial diversity and excessive number of racial stereotypes prevalent in many video game titles, (Shliakhovchuk \& García, p. 47-48). This has helped them recognize the inherent problematic values and assumptions that are built into the games design. Additionally, playing video games has helped players increase their cultural expertise, acquisition of cultural knowledge and communication skills, as well as increasing players' level of self-awareness, understanding, and cognitive and cultural empathy towards others, (Shliakhovchuk \& García, p. 48-49). These direct impacts of video games on a player's cultural perspective speaks volumes to their efficacy in promoting both their own unique subculture and incorporating ones previously unfamiliar to them. This also plays an enormous role in the production of gaming capital, as the acquisition of knowledge and skill and, in turn, sharing them with other player, is an essential aspect of producing gaming capital.

Now, shifting focus to social features of video games, the production of social capital is very unique compared other forms of social interaction due to the lack of face-to-face interaction in most gaming settings. Often times, as Jane McGonigal points out, players don't even have to 
directly interact in-game with one another to get the social capital they are looking for. In her review of Palo Alto Research Center's study of 15,000 World of Warcraft players, she notes that over $70 \%$ of players actually perform missions alone or with NPC support character. Some of this has to do with the shift in the culture of WoW, which I will discuss in the analysis section, but a lot of the rationale behind this type of play is the concept of ambient sociability. McGonigal points out from this research that many players "expressed that even though they were not directly interacting with other players, they enjoyed sharing the virtual environment with other players, expressing a high degree of "social presence," (McGonigal, p. 90). This is the epitome of ambient sociability, having social connection without physically interacting with the other people in the space. I would liken this relationship to someone sitting in a park or coffee shop, enjoying the presence of other people in the same setting. Players don't feel alone in these settings because they recognize other individuals engaging the same or similar actions in game, producing a common understanding that is shared between all players. McGonigal does note that social bonds are not established in a way that produces significant social capital, but it does create connection and social expansiveness that allows for the production of social capital if the individual wants it. She also points out that this is a great tool for introverts who struggle with socialization, allowing for them to gain access to casual social connections while training their brains to experience social interactions as rewarding experiences where they normally would not, (McGonigal, p. 90-92). Video games do have a more direct influence on a player's socialization and their production of social capital. For example, in their analysis of video game players, Shliakhovchuk and García find that of the behavioral impacts of video games, the highest noted by players is increased pro-social behaviors like helping others, increased sensitivity towards “out groups," and increased communication ability, adaptability and resourcefulness, 
(Shliakhovchuk \& García, p. 47-49). These are essential facilitators for the production of both social and cultural capital, as it creates a more diverse and supportive perspective for players when interacting with others in-game. Now that I have established the more general impacts on cultural and social capital in playing video games, I need to briefly discuss how gaming capital comes to be in these contexts before I move on to more in-depth analyses of these features.

As Walsh and his co-authors point out, video games are "cultural objects, consisting of machine and a game simulated in software that finds significance in the history and materiality of it, (Walsh et. al., p. 2). Video games, as well as the culture and socialization surrounding them, are produced and facilitated by a combination features that must all work together effectively to create an enjoyable gaming experience. Walsh and his co-authors point to two primary features that help produce the gaming culture and the capital that comes with it, which are unit operations and procedure rhetoric. Unit operations are, "modes of meaning-making that privilege discrete, disconnected actions over deterministic, progressive systems. This means that the moments of play are equally as significant as the game in its entirety. The individual unit operations may take the form of actions, movements, and/or configurations," (Walsh et. al., p. 3). The individual actions and decisions made the player have meaning behind them, which in turn gives value to the game itself. Additionally, procedure rhetoric supports this development of meaning and refers to, "the ways VGs enact ideology in their computational structures by using rule-based representations and interactions to convey meaningful and persuasive argument in the code of games, which is enacted through gameplay and may be accepted, negotiated, or rejected by the gameplayer," (Walsh et. al., p. 3-4). These are the rules and guiding features of video games embedded in the code that dictate what is and isn't an acceptable aspect of the culture surrounding the game and the actions that players take. The combination of the microlevel 
actions of players and the negotiations between them and the software coding of the game establish the recognizable subculture of each game. For example, Call of Duty has had a long history of implementing overpowered and at times game-breaking features into their games that never cease to enrage the community, leading to debates on how to fix the issues. Without input from players, these problems would never be adjusted but this push and pull between player and developer guides the production of a unique culture within Call of Duty. As an additional tool for supporting the development of the subcultures of specific games is the use of "paratexts." These are mediums like gaming magazines, walkthrough guides or even instructional YouTube videos that help spread information about popular topics within the field of video games as well as trick and tips that help make the game easier for the average players. These are essential for expanding the video game's culture, and the production of gaming capital, past the bounds of the virtual world. I will discuss the concepts of paratexts in the analysis section more in-depth, as they play an essential role in the production of culture and tools for players to increase their position within the social structure. Now that I have expounded briefly on what these forms of capital look like in video games, I can now shift focus to looking at specific examples of the production of economic, social and cultural capital in the context of video games. following that analysis, I will break down how they culminate into gaming capital and what these forms of capital mean for the larger context of social interaction outside of the game. 


\section{Varying Forms of Capital in Video Games \\ Production and Conversion of Economic Capital in Video Games}

To begin my analysis section, I will first breakdown some of the primary ways in which individuals can produce economic capital through the video game industry, ranging from game testers to professional eSports players and streamers. Following this I will look at the explosion of "loot boxes" and other forms of microtransactions, how they are creating expansive in-game economies, and the use of third-party trading websites for games like Rocket League. These features are a phenomenal example of the varying ways in which economic capital plays a role in the development of social and even civic interaction within the context of video games. They also provide my research with examples of how actions in game can increase an individual's economic capital in the real world.

With the exponential development of the video game industry, there are a variety of new job and money-making opportunities available to those involved in the community. One of the most basic and easily accessible ways to make money in this industry is as a game tester. According to his article, Miller states that Quality Assurance Testers are at the base level of game development and their testing process involves analyzing software and design features, looking for flawed coding, game bugs, and testing a variety of character builds and play styles. This is in an effort to assure that the player base is receiving the highest quality and most enjoyable game play experience. Most QA testers make around \$15-20 an hour and is a great entry level position for the industry while still impacting the important social and cultural features within the game. Moving up the chain, the next position is Game Tester, which is similar to a QA tester but instead of testing software aspects, their job is primarily focused on actually trying to break the game by pushing the boundaries of the hardware and coding. This 
involves trying to find loopholes in the game's code, as well as trying to do complex maneuvers and tactics that may not be accounted for in the game's design. The purpose of this job is ensuring that the game will not have severe technical issues upon release and essential for the effectiveness of the game's performance, (Miller, par. 4-9). Both of these jobs involve the structural and technical aspects of game development, helping to stabilize the social environment within the game. They are great examples of the community actively facilitating a more engaging and positive gaming experience.

Another vein of economic capital production available to players is through the professional eSports/streamer route. Both of these avenues provide extensive opportunities for earning money like winning tournaments, gaining Twitch/YouTube channel subscribers and being offered sponsorships like a traditional sports athlete. Along with the monetary gains available in these industries, eSports players and professional streamers have a variety of opportunities for both community engagement through their platforms as well as a much heavier influence on game development, (Miller, par. 10-14). For example, many of the professional eSports leagues like Overwatch and Call of Duty have players associations that work with game developers to make adjustments to weapons, game modes and maps in an effort to better balance the game and create a more enjoyable experience for the whole community. These jobs clearly have an influence at the social and cultural level of the video game environment and provide a litany of situations in which production of economic capital is fairly simple. Another, less popular area of economic capital production in video games is game review sites like Banatic or InboxDollars. These sites pay players, mostly in the form of gift cards or cryptocurrency, to play and review new games in an effort to increase popularity of the game. Most of the games they review are from independent developers who are looking to get their name out there and 
facilitate the production of a larger player base. All of these job opportunities are great examples of how individual players can produce their own economic capital through video game play. I will now shift focus to the development of in-game economies in games like Rocket League through the advent of microtransactions and third-party trading sites.

Microtransactions are a relatively new aspect of video games and are largely replacing the traditional revenue streams for game developers. Microtransactions fall into two major categories, cosmetic and "pay-to-win," which I discussed in chapter 1. As a quick reminder of these concepts, cosmetic microtransactions refer to "any situations in which spending additional money leads to aesthetic change within the game but no in-game advantage," (Zendle et. al., p. 2). Pay-to-win microtransactions on the other hand are "any situation in which players are able to exchange real world money for something that increases their change of in-game success," (Zendle et. al., p. 2). In terms of this discussion, I will avoid discussing the pay-to-win model, as it is largely received by the community as an unfair advantage for players with access to inherited economic capital and most major game developers have steered away from using them for this reason. Instead I will focus on cosmetic options, which include things like skins and emotes (i.e. dance moves or actions that a player can use as a celebration or taunt in-game) in Fortnite or Painted car skins or wheels in Rocket League. These items, as they must be purchased with real world money, hold inherent economic value to the player. Along with their economic value, the ownership of these often-rare cosmetic items confers status and prestige within between players. Players with rare skins are seen as high-level players, often referred to as "sweats." The term sweat comes from the assumption that the player is so focused and playing at such a high level that they are literally sweating as they're playing. It is both a term to indicate the prestige of the player, but it is also used derogatorily when players are upset about being 
placed in a lobby with a player of a higher caliber. These skins obviously hold have no impact on the performance of the player, but they tell the other individuals in the lobby that the player in question is likely better than them.

As I mentioned above, the fact that these skins must be purchased with real world money makes them engrained with economic value to the player. In the case of games like Rocket League or Counter Strike: Global Offensive (CS:GO), these items are attained through a loot box system. Loot boxes are essentially like slot machines with items in them; players earn the loot boxes through leveling up in-game and require purchases of keys to open said boxes. The algorithm used for these boxes, and many other looting generating systems in video games is known as a random number generator or RNG. In an RNG system, more common items have a high likelihood of coming up and more rare ones have a lower likelihood, giving the rarer ones a much higher value to the player. Something fascinating about loot boxes that Zendle and his coauthors point out is that of the $\$ 140$ billion in revenue for the video game industry in 2018 , around $\$ 30$ billion came exclusively from loot boxes, with that number rising every year, (Zendle et. al., p. 3). Because of their economic value, players seek out these rare items in any way they can, often using in-game or third-party trading services to access them. In the case of Rocket League, the game developers implemented a trading platform in-game in which two players enter a menu, place what items they wish to trade, with both players needing to press accept before the trade can go through. Trading in Rocket League has become increasingly more complex with the development of new skins, goal explosions (i.e. colorful celebration after a goal is scored) and wheels. These cosmetic features illicit positive emotional responses from players, through the use of bright colors and lights. These operate in much of the same way as stadium features like fireworks or confetti when a major sports team scores. These items can be 
their base color or painted with a variety of colors which would be of higher value. The value of these items also shifts over time, making the trading process challenging to new players. In an effort to alleviate some of these barriers, third-party websites like RLExchange have been created to provide pricing lists, tips on how to trade effectively, and forums for players to post about their trade offers and find potential suitors. Some tips that RLExchange offers include learning item values by reviewing their price list, regularly posting on the forums and keeping them up in order to find the best deal, having confidence in the exchange itself, and avoid scammers at all costs, (RLExchange, par. 12). By trading their rare items, this process allows for individuals to make real world money from other players. Most game developers have said they do not support this type of trading economy, but many players still offer their items on thirdparty sites in exchange for real money or even keys to open more loot boxes. This process is a fascinating example of an in-game economy not only expanding internally, but also has real world economic value available for players. It highlights the effectiveness of producing economic capital in video games. In the next section, I will breakdown more specific examples of video game cultural capital, how the cultures of some of these games has shifted over time, and a great example of real-world cultural artifacts finding a home within a virtual world.

\section{Cultural Capital in Virtual Worlds}

As a starting point for this portion of my chapter, I need to reassert the traditional understanding of video game culture and how this perspective has changed over time. As I have mentioned at length in this thesis, the traditional view of the prototypical video game player is an anti-social nerd with addictive and violent tendencies. The toxic and masculine culture surrounding video games that I discussed in chapter 1 is one of the most prevalent features in the conversation surrounding video games and is something game developers have worked tirelessly 
to change. One of the major reasons for this culture persisting is the lack of a feminine perspective within game development, something Shaw alludes to in her research. She notes that to better combat this negative and abusive culture is "not to shift a focus to female gaming groups... What is necessary is a critical reexamination of the place of women and girls in those spaces of gaming culture that have been traditionally defined as male," (Shaw, p. 409). What Shaw is positing is an inclusion of women in the story and character design, giving female characters more diverse characteristics that are more in-line with the modern woman. Women are often displayed as either helpless, "damsel in distress" types or as cold, hardnosed villains, further perpetuating an inaccurate representative of women in video games. Including women in the development process can help remove some of these outdated tropes and stereotypes.

Toxic masculinity and abusive behaviors are not the only feature of video game culture however and are largely being eradicated from the environment with every passing day. The culture of video games in the modern context is far more diverse, producing a subculture that is still deeply intertwined with the larger pop culture umbrella. As Shaw points out, gamers are often designated by specific styles of dress, unique lingo, and a strong sense of solidarity and unity within the community. Much of the verbal interactions and ways in which they present themselves in terms of clothing and dispositions are in reference to specific games which are not widely understood outside of the community, (Shaw, p. 409-411). This creates a very tight knit bond between players who otherwise would not be together, speaking to the effectiveness of video games at facilitating the production of bridging social capital, which I will discuss in the next section. Additionally, the video game subculture is inherently part of the broad societal culture in modern society, as it has become interrelated with meme and social media culture. So many popular memes in today's society find their origins in video games, from funning NPC 
interactions to clips and videos of player freak-outs, the two subcultures are complementary of one another. A great example of video games and meme culture becoming intertwined is the "Press F to pay respect" meme that has become increasingly popular on platforms like Twitch, Twitter and Reddit. The phrase comes from the Call of Duty: Advanced Warfare campaign in which the play enters a cutscene (brief cinematic that helps provide depth to campaign missions) where your playable character attends the funeral of a fallen soldier from your unit. In the cutscene, the player receives a prompt when walking up to the coffin that says, "press F to pay respects" ( $\mathrm{F}$ is the action key for many computer games). The phrase became viral on Reddit forums related to video games and is now being used a common statement in Twitch chats and Twitter feeds when someone makes a mistake or has something unfortunate happen to them. Chat members will follow up the streamers mistake with a simple " $F$ in the chat," denoting that they noticed the problem and are poking fun at the individual that it happened to. The phrase itself is now an integral aspect of meme culture, increasing the popularity of memes overall. As memes from games become more popular, so to do the games themselves. Video games, therefore, are not a counterculture, completely removed from the larger perspectives of society and actively working against the notion of a widespread culture. Video games are instead being co-opted by pop culture, becoming intertwined with it so much so that video games are an enormous portion of the cultural products on other forms of digital media like Twitter and Reddit.

Shaw also points out that when we define the culture of video games, the first two concepts that come to mind are immersion and interactivity, which was a primary focus of my second chapter. When analyzing these features, Shaw quotes Walker's work who states that they facilitate, "an active, complex and multilayered form of escapism involving high action face- 
paced media," (Shaw, p. 412). These features lead us to develop a cultural appreciation for ingame items, lore and just general activities. For example, in Animal Crossing, players collect resources and rare items and have two options for what to do with them. Players can either donate them to the in-game museum which can be accessed by any player and allows other to appreciate their cultural and artistic significance in-game. Players can also just simply sell them in Tom Nook's (main NPC in the game) store for in-game currency, (GlassCaseInPoint, par. 23). These two options highlight the decision that players must make in terms of the type of capital they are looking to produce in-game. If player sells them to Tom Nook, they can increase both their economic capital (for obvious reasons) as why as their symbolic capital as the increase to their financial stores allows them to access more rare items, house designs, etc. that display their status in-game. If the player decides to donate the items to the museum, their cultural and social capital are increased in the sense that they are increasing access to culture for the server and providing more opportunities for social interaction between players while enjoying the museum displays. Another great example of a balance between economic and cultural capital in video games from the same article is in reference to the Art Dealer in the Witcher 3 title. During the Art Dealer process, players must first answer a series of art-related questions (these questions are pertinent to real world art and the player must have exterior stores of cultural capital in order to answer them) in order be allowed to engage in deals. As the article states, "this scene attempts to reconcile the tensions between economic and cultural capital. Geralt (the player-character) is able to invest soundly in Fine Art only because he proves that he can appreciate it. The Art Dealer recognizes Geralt as a figure of expertise and is therefore happy to sell to him - the exchange is one of Cultural Capital as well as Economic Capital," (GlassCaseInPoint, par. 4-6). These features in both Animal Crossing and the Witcher 3 are excellent representations of the 
importance of cultural capital, both inherited and learned through play, when engaging in online gaming. They facilitate not only a cultural understanding of the game itself, but also exterior forms of cultural previously unknown to the player. Next, I will look at how WoW has shift over its lifetime from almost entirely promoting socialization and cooperation to allowing for more solo queue options to players, which has shifted the culture surrounding the game itself.

World of Warcraft has, since its infancy, been primarily focused on socialization and community building between players. With the explosion of guild memberships and the thousands of team-based dungeons and raids, WoW has always been about bringing players together to work to achieve common goals. This, however, has hindered many introverted, antisocial players from being able to achieve the same level of social and symbolic capital as those who are more social. In an effort to help combat this, Blizzard has over the years implemented a variety of solo play options to players that still mimic many of the team-based objectives in-game. WoW has always been known as a "possibility space" as Braithwaite calls it, that enables some actions and precludes others. They do not only create the experience of play; they also construct the meaning of the game itself. Therefore, changing the rules of the game changes the possibility space, (Braithwaite, p. 5-6). The author compares this to public policy in government decisions in that the game developers set the social rules of the environment, they create the system that society operates on. We see this with the use of Group Finder mechanics and guild structures, almost forcing social interactions on players in order to move forward ingame. Braithwaite, in quoting Simon's work, refers to this as designed sociability, meaning forms of sociality or social structures that have been hardwired and coded by the design choices of developers, (Braithwaite, p. 5). This does not mean that the designers dictate how and when players interact, but rather the social structure of the game provides or limits certain 
opportunities for social engagement and in doing so shapes how players interact with one another and the relationships that form that interaction. In an effort to minimize the requirements for socialization, WoW has begun to design raids and missions that do not require queuing up with other players to complete them. A great example of this is the Proving Grounds system which allowed players to use NPCs that they recruited from around Azeroth as teammates as opposed to real players in-game. It allowed players to operate like a general of sorts, directing their squad of computer-generated players against the monsters and mission boss. Another good example that Braithwaite provides is the development of the solo queue matchmaking for both $\mathrm{PvP}$ and PvE events. This allows players, much like the Group Finder mechanics, to find players after they load up a mission through an autogenerated matchmaking system, filling out their team for them, (Braithwaite, p. 7-10). These features discussed have drastically shifted the culture of WoW, and the production of cultural capital, away from socialization and engaging with other players towards a more automated system with minimized contact between players. This is inherently positive for the community in that all the old features for finding guild members and engaging in activities are all still in place but there are new, less social ones that allow introverted players to enjoy the same experiences as everyone else. This expands the community and culture surrounding the game itself. In the final portion of my discussion of cultural capital in video games, I will look at the Uncensored Library, a Minecraft server entirely dedicated to providing banned scholarly and journalistic texts to anyone who wants them.

The Uncensored Library is a relatively new Minecraft Server, developed in 2018 by the German branch of Reporters Without Borders. The purpose of the server is to provide articles, scholarly journals, etc. that are banned in countries like Russia, Mexico and North Korea to anyone who accesses the server. The beauty of this design is that Minecraft is accessible to 
anyone with a computer, even the those of the lowest quality, and is not banned in any country, creating a unique loophole to the journalist oppression around the world. The world itself contains a massive virtual library, designed with many of the same structural features as the New York Public Library and contains thousands of banned texts from around the world. When you enter the library, there is massive map containing all 180 countries on Reporters Without Borders' Press Freedom Index with their corresponding ranks, (Maher, par. 2-12). This is such effective symbolism for the players on the server, giving them visible indication of the types of authoritarian limitation of media that still happens all around the world. The server has members from over 175 countries and the website version has had visitors from all but 10 countries in the world. It has actually received so much attention that it experienced a total crash three after launch forcing the moderators to upgrade their capabilities to support the number of players. The server itself is so large it requires in-game teleporters for players to travel between different wings of the library. It has dedicated rooms for the largest violators of press freedom like Russia and Mexico and murals of some of the journalists who have been victims of violence like Jamal Khashoggi from Saudi Arabia, (Maher, par. 9-10). The scale and scope of the server alone speaks to the possibility for cultural and social capital production, having potentially thousands of players interacting with one another, debating various political and social issues and bonding them together over a shared interest in these topics. It also provides extensive opportunities for players to expand their cultural knowledge and, in turn, provide the same to new members. It is a fascinating example of the interplay between video game culture and the more general culture of human society. In the next portion of my analysis I will look into the defining features of video games that facilitate social capital production and what that means for the larger context of socialization within the online gaming community and to society as a whole. 


\section{Socialization Producing Capital Through Gameplay}

Video games over the course of the last 20 or so years have become inherently social experiences that provide a variety of positive features to players when trying to build their social capital in the virtual setting. As Molyneux and his co-authors point out, "players in these games go beyond social interaction to participate in shared tasks and objectives within the virtual world where they meet. Social media may be considered a third space where people can meet and interact, but they do not provide the same kind of alternate world that is the setting of many video games. Within these worlds, players become part of social hierarchies that frequently collaborate to accomplish game objectives," (Molyneux et. al., p. 383). Socialization is now an integral aspect of game design and is in most cases, the primary motivator for why individuals play video games. It creates an environment of meaningful play in that the actions taken by the player not only have significance to themselves, but also the other individuals they are playing with. Another positive feature that Molyneux and his co-authors note is that these games, because of their diverse player base and almost necessary socialization, expose players to a variety of cultural and political perspectives which actually make us more considerate and empathetic to the struggles of these groups, (Molyneux et. al., p. 385). One of the major outcomes of these authors' research is that social capital produced through video game play is actually related to an individual's frequency of civic engagement. They find that there is not a direct correlation between the two, but rather that those individuals who play video games have a much easier time in developing the skills needed to participate in civic engagement and are more likely to use them, (Molyneux et. al., p. 394). This interconnected relationship between social capital produced in video game play and willingness to engage civically with other is just one 
great example of the positive influence that socialization through gameplay has on the player. It facilitates positive social interaction both in-game and in real world settings.

What are some of the key reasons for why individuals want to play games with others? As Lee points out in his article, leisure activities like playing games have been proven to improve the overall connectedness between players and improve their overall wellbeing, (Lee, par. 5-6). When looking at social capital, there are two primary forms in which can manifest, bonding social capital and bridging social capital. Bonding social capital refers to strong ties like family or close friends that provides emotional or substantial support to the individual. Bridging social capital on the other hand is found in weak social ties like long distance friends and provides valuable information outside of one's close network but does not translate to substantial support, (Lee, par. 24-27). When looking at Yee's analysis results, we see that playing with local ties and distant ties (both friends and family) produces significant bonding social capital for the player whereas playing alone or with exclusively online friends does not. Additionally, all except playing alone were found to increase a player's bridging social capital. He also found that both playing alone and with local ties was directly related to higher levels of civic engagement, (Yee, par. 27-28). These results highlight the diverse range of social capital that can be produced through video game play and how varying levels of social connection can influence that. Video game clearly have a direct and significant impact on social capital production.

I now need to establish the process through which players acquire and utilize the social capital available. According to Reer and Krämer's research, there are three underlying factors that influence social capital acquisition. The first of which is physical proximity, which refers to the availability and accessibility of other for interaction and helps foster social affiliation and enhances the chances of individual's forming friendships. The next is social proximity, which is 
the closeness of one's social network and produces social attraction and affiliation. Finally, familiarity refers to social psychological notion that humans feel comfortable and drift towards things that feel familiar and promotes the acquisition of social relationships, (Reer \& Krämer, p. 180-181). These three factors are the defining aspects of how we produce and utilize social capital and they provide my analysis with a better perspective on how social interaction can and does take place in these settings. Reer and Krämer's research is focused on determining how these factors influence socialization in the context two unrelated games, of which I will focus on their evidence regarding World of Warcraft. In the case of WoW, the authors find that some of the direct effects of the game are a positive connection between offline activities between players and the frequency of their communication. Also found a significant link between offline activities and their ability to build bonding social capital. Being an administrator of a clan/guild in WoW was also correlated with communication frequency and bonding social capital. In terms of indirect effects, the authors found that players who meet offline, regularly play together and are involved in the administrative process of a guild are all positive influencers on social capital acquisition, (Reer \& Krämer, p. 184-185). The evidence provided regarding WoW socialization proves the ability of these video games to facilitate the acquisition social capital in its players as well as the utility of that social capital going forward in possible community interaction in the future. Socialization in video games, as I mentioned above, is integral to the success of the game itself, as players regularly seeks opportunities to engage with other players. Finding ways to promote social capital production within their games will dictate how and why individuals play these games. In the final section of this chapter, I will look at how these three primary forms of capital coalesce into a form of symbolic capital unique to video games, gaming capital. I will 
also look at how certain design features, along with the input of the community, further stabilize the hierarchy within these games, promoting a drive for prestige and status in its player base.

\section{Gaming Capital}

When attempting to understand the production of gaming capital, we must look back at Maher and his co-author's definition of the field, echoing Bourdieu's conception of it, which is a "system of objective relations of power between social positions which are limited to a system of objective relations between symbolic points," (Mahar et. al., p. 8). This conception of the social structure views society, not as a singular unit, but rather as an interwoven map of competing and complementary fields of social interaction. When we look at virtual field of video games, the same notion must be incorporated. Video games are an essential aspect of the larger social and cultural perspectives in our society and ignoring that fact would make a view of them shift to a space free of consequences or limitations which is simply not the case. This is where gaming

capital comes into play which, as Toft-Neilson and Strøm Krogager allude to, is a unique form of capital that facilitate social contracts between players through the contact between one another and the sharing of knowledge, skills, and cultural perspectives within the community, (ToftNeilson \& Strøm Krogager, p. ). The sharing of knowledge, and to a larger extent, the stores of capital available to players, helps to expand the culture and social networks of these games to a larger audience, incorporating them into community. It is through this interaction and exchange of knowledge and resources that gaming capital manifests. The development of gaming capital, which as I mentioned above, is the process in which this specific social field produces symbolic capital through the accumulation and application of the other three forms when engaging in game play behaviors and actions. Symbolic capital denotes an individual's prestige, status and place within the hierarchal structure of a social space. It facilitates a general perspective of others in 
the social space and one of the best ways that this can happen in the context of video games is through the use of ranking systems and group hierarchy. As it was the primary focus of my third chapter, I will not go too in-depth in the discussion hierarchal structures, but I feel it is necessary to reaffirm their role in establishing our place identity in the context of a social field. The ranking systems I discussed in chapter three (i.e. Elo and TrueSkill for a competitive hierarchy and Social Score and commendations for a social hierarchy) play an integral role in in how we perceive ourselves in the relation to others in the same space, comparing our accomplishments to that of other individuals, seeing where we find our place in the system. Additionally, these systems are largely meritocratic, meaning that the level an individual receives is dependent on their performance in-game and is meant to accurately balance multiplayer lobbies, so players are matched up against equal competition. The intention is for players to utilize their stores of social and cultural capital, meaning the skills and relationships they've attained through play, as tools for increasing their position. By learning new strategies, developing a better understanding of the maps and gameplay, and regularly playing with the same group of friends, players can become objectively better at the game and increase their position within that hierarchy.

However, there are a variety of unsanctioned methods by which players increase their position without utilizing their skills and competence. What I am referring to is the increased prevalence of modding and hacking that has caused a litany of issues for both developers and players. Each of these terms denotes a different action that can overlap in a number of ways and all can fall under the banner of cheating. Thanks to a forum post on GameFaqs.com, I have two great definitions to help create some distinctions between the two. Modding refers to "glitches, modified textures, or improved gameplay mechanics," (GameFaqs.com, par. 3). These are outside of the conceive or intended design of the developers and can in some cases cause major 
issues to the game servers as a whole. Hacking, on the other hand, refers to an individual "manipulating servers (or sometimes taking down servers), game files, and even produce illegal digital copies of a game without ever being traced by the developers of the game or company," (GameFaqs.com, par. 4). The major difference between the two here is that hackers create the mods and make illegal edits to the game's design or mechanics and the modders just utilize them. It is a system in which individual's, who are attempting to circumvent the current system of hierarchy, actually create their own trickle-down system of authority and hierarchy. The intention of these mechanics is simple; players who are unable to achieve the rank, level or social position within the game that they believe they deserve utilize them to increase that position. Modifications or hacks are related to capital usage however, as many of them are not free. Players use their stores of inherited or accumulated economic capital to purchase these tools, which again speaks to the unfair advantage they can have access to due to the disproportionate access to economic capital.

The final aspect of the production of gaming capital that I must discuss is something I touched on at the beginning, which is paratexts. Paratexts are aspects of literary work that is outside the bounds of main authorial text but provide a frame of reference for understanding the work itself. For instance, a book's cover is not part of the main written aspects of the book itself, but it provides an inclination of the content it will contain and draws in new readers. When looking at paratexts in the context of video games, there are three primary forms that facilitate the production of gaming capital outside of the virtual setting. The first of these paratexts are gaming magazines which, according to Märyä's research, have a variety of roles for promoting and developing new forms of video games. These magazines place importance emphasis on the educational and cultural role that they play in the development of the player base, as well as 
eliciting and cultivating both the value of the game itself and what makes a good or bad game, (Mäyrä, par. 9-10). Gaming journalism helps to produce gaming capital by exposing players to new content, hardware, possible tips and tricks for advancing in the game, all of which are examples of gaming knowledge (i.e. gaming capital). The second form of paratexts in the video game industry is strategy guides. These are similar to gaming magazines but focus less on news stories, new content, etc., and instead provides in-depth walkthroughs, strategies and overall gameplay basics for the player in an effort to educate them on the best ways to be successful ingame. One of the best features that Mäyrä points out in these strategies guides for creating the most supportive system of educating players is the separation of spoiler content from the main bulk of the guide, (Mäyrä, par. 11). This allows players to still get the same important information about how to get past difficult levels or learning about the lore of the game without ruining the secret or unique content of the game itself. This system minimizes the consequences of trying to accumulate varying forms of capital in online gaming. The final form of paratexts, add-on devices, is quite different from the other two in that it is an actual piece of hardware that changes the dynamics of the game. These can and do fall under the category of mods and as many developers argue, shorten the lifespan of the game by minimizing the challenge presented to the players, (Mäyrä, par. 12). It reduces the ability of the developers to guide the socialization and culture acquisition in the ways that they want, allowing players to avoid many of the barriers put in place by the game's designers. Regardless, paratexts clearly provide players with additional sources for producing and accumulating cultural, social and symbolic capital by using their stores of economic capital. They may not have as detrimental of an impact on the integrity of the game as hacks and mods do, but they still allow players to increase their position in the social hierarchy without having to work on just simply being better at the game. They can learn 
strategies and tips without having to perform a trial and error analysis with every action. Overall, this section has shown how the use of the other three primary forms of capital help facilitate not only a general sense of prestige and status through the production of symbolic capital, but also how video games, through their unique subculture, have produced their own form of symbolic status, gaming capital.

\section{Conclusion}

To conclude this chapter, and my thesis as a whole, I would like to reaffirm what has been discussed and how, through the evidence provided, I have shown that video games truly are an emerging and effective setting for social, political and economic interaction. In the first chapter, I established the traditional perspective of video game culture as a toxic and abusive environment, as well as the more contemporary understanding of the overarching norms and values associated with video game play like increased pro-social behaviors, positive cognitive development, cooperation and problem solving. These norms and values, which culminate into an individual's habitus, impact how, when and why we interact with others. The structure of these games makes the process of developing a habitus easier through a lack of limitations on the access to a variety of cultural, social and political perspectives, which help to shift our own understanding and disposition towards other actors in the setting. Chapter one helped to establish what we perceive video games to be and what an individual's, and the community's, habitus can be.

In chapter two, I focused on the virtual social field of video games and how the structure and design, along with input from the community at large, influences the social interactions that take place within the game. This chapter focused heavily on the importance of immersion and interactivity when facilitating a positive social environment as well as the role of the sensation of 
flow on how we conceptualize the setting of video games. Mihaly Csikszentmihalyi's flow theory provided my research with evidence that an active and dynamic virtual setting like video games mimics the many of the same features as real world socialization. I also highlighted that design and structure of these games creates an environment that reproduces our real-world norms and values as well as facilitating the development of new ones. These norms and values can and do related to our understanding of socialization, political discourse and economic interaction, as well as a noticeable impact on an individual's habitus, both within the game and in the real world. The chapter analyzed the role that the eSports industry, from the executives of major game development companies all the way down to the individual player, in the development of this social space and the community contained in it. I also looked at how, through the structural design of the World of Warcraft server, Blizzard has been able to make one of the most social and civically minded video game environments ever created. Both of these examples highlighted the role that structure and setting play in our ability to socialize with one another.

In the third chapter, I continued my analysis of the structure and design of these video games but too a more in-depth look at the importance of social and political hierarchies within them. Again, I looked at the role that the eSports industry has played in developing effective matchmaking ranking systems that place players within a tier system based on their apparent skill level. This system produces accurate, meritocratic hierarchy for players, helping them to understand their place within the social environment and what they can do to increase that position. Additionally, I examined how the guild system in WoW operates in much of the same way as political systems in the real world do, with complex hierarchies that facilitate cooperation, teamwork and civic engagement in its members. These structures, while largely authoritarian in nature, provide numerous opportunities for players to engage civically, through 
guild forums, elections of guild leadership, and shared economic objectives through contributing to the overall resources of the guild. Many of them operate in a manner similar to hybrid regimes in the modern context, with centralized authority supported by various democratic institutions. These systems were found to have numerous positive impacts on a player's ability to socialize and think civically.

In my final chapter, I took Bourdieu's four major forms of capital and applied them to the context of video games as well as analyzing how they work in tandem to produce an individual's gaming capital. The process involves a give and take of acquiring and utilizing these forms of capital through various mediums both internal to the game world and through external producers of capital. Through the development and application of the varying forms of capital, the traditional understanding of the video game subculture as anti-social, aggressive nerds has been replaced with a new perspective that sees video games, not as counterculture, but rather something that pop culture has co-opted and incorporated into the overarching cultural perspective of society. Prestige/status, social skills, cultural knowledge, and access to economic capital within the context of video games can and does impact our access and utilization of the same forms of capital in the real world. It provides opportunities for the development of capital without the same restrictions and limitations that traditional society places on individuals.

Overall, this thesis has effectively shown the role that a video game's community, structure, and its varying forms of capital have on producing a supportive environment for socialization and civic engagement. 


\section{WORKS CITED}

Adinolf, S., \& Türkay, S. (2018). Toxic Behaviors in eSports Games: Player Perceptions and Coping Strategies. CHI PLAY'18 Extended Abstracts, 365-370.

Anderson, C. A., \& Bushman, B. J. (2001). EFFECTS OF VIOLENT VIDEO GAMES ON AGGRESSIVE BEHAVIOR, AGGRESSIVE COGNITION, AGGRESSIVE AFFECT, PHYSIOLOGICAL AROUSAL, AND PROSOCIAL BEHAVIOR: A Meta-Analytic Review of the Scientific Literature. American Psychological Society, 12(5), 353-359.

Bailey, K. (2018, April 26). Five Social Benefits of Introducing Minecraft to Your School. Retrieved from https://education.minecraft.net/blog/five-social-benefitsof-introducing-minecraft-to-your-school

Bonaiuto, M., Mao, Y., Roberts, S., Psalti, A., Ariccio, S., Ganucci Cancellieri, U., \& Csikszentmihalyi, M. (2016). Optimal experience and personal GROWTH: Flow and the consolidation of Place Identity. Frontiers in Psychology,7. doi:10.3389/fpsyg.2016.01654

Bourdieu, P. (1984). Chapters 2-4. In Distinction: A social critique of the judgement of taste (pp. 99-250). Cambridge, MA: Harvard University Press.

Bourdieu, P. (n.d.). Forms of Capital. Handbook of Theory and Research for the Sociology of Education, 1-13.

Bourgonjon, J., \& Soetaert, R. (2013). Video Games and Citizenship. Purdue University Press, 15(3), 1-7. 
Bown, A. (2015). Chapter 2: Unproductive Enjoyment: A Culture of Distraction. In Enjoying It: Candy Crush and Capitalism (pp. 26-38). Winchester, UK: Zero Books.

Braithwaite, A. (2015). WoWing alone. Games and Culture, 13(2), 119-135. doi:10.1177/1555412015610246

Buckley, K. \& Anderson, C. (2006). Chapter 24: A Theoretical Model of the Effects and Consequences of Playing Video Games. In 1170511295877803190 C. A. Anderson (Ed.), Playing Video Games: Motives, Responses, and Consequences (pp. 363-378). Mahwah, NJ.

Call of Duty Staff. (2018, March 19). Stepping Up Your Social Score [Web log post]. Retrieved from https://www.callofduty.com/blog/archives/step-up-your-socialScore

Call of Duty Staff. (n.d.). League Play. Retrieved from https://callofduty.fandom.com/wiki/League_Play

Call of Duty Staff. (n.d.). Prestige Mode. Retrieved from https://callofduty.fandom.com/wiki/Prestige_Mode

Carbonie, A., Guo, Z., \& Cahalane, M. (2018). Positive Personal Development through eSports. In Pacific Asia Conference on Information Systems (pp. 1-6). Association for Information Systems.

Chen, J. (2007). Flow in Games (and everything else). Communications of the ACM, 50(4), 31-34. 
Cheng, M., She, H., \& Annetta, L. (2014). Game immersion experience: Its hierarchical structure and impact on game-based science learning. Journal of Computer Assisted Learning, 31(3), 232-253. doi:10.1111/jcal.12066

Chuen-Tsai, S., \& Hao, W. (2011). Game Reward Systems: Gaming Experiences and Social Meanings. In DiGRA 2011 Conference: Think Design Play (pp. 1-12). Authors \& Digital Games Research Association DiGRA.

CivCraft: Build More Than Just Blocks [Web log post]. (n.d.). Retrieved from https://www.civcraft.co/

Cote, A. (2020, August 21). Here's what it'll take to clean up eSports' toxic culture. Retrieved from https://theconversation.com/heres-what-itll-take-to-clean-upesports-toxic-culture-143520

Csikzentmihalyi, M. (1997). Finding Flow. Psychology Today, 1-7

Dalisay, F., Kushin, M. J., Yamamoto, M., Liu, Y., \& Skalski, P. (2014). Motivations for game play and the social capital and civic potential of video games. New Media \& Society, 17(9), 1399-1417. doi:10.1177/1461444814525753

Deen, P. D. (2011). Interactivity, Inhabitation and Pragmatist Aesthetics. The International Journal of Computer Game Research, 11(2).

Dennis, A. (2020, March 20). Microtransactions and their future in gaming. Retrieved from https://medium.com/ringnetwork/the-future-of-gaming-howmicrotransactions-are-disrupting-the-industry-c7f5bab1081b

Dietz, T. L. (1998). An Examination of Violence and Gender Role Portrayals in Video Games: Implications for Gender Socialization and Aggressive Behavior. Sex Roles, 38(516), 425-440. 
Espinosa, D. (2017, January 16). Video game rating systems: Is Elo hell real?

Gailey, C. W. (1993). Mediated messages: Gender, class, and cosmos in home video games. The Journal of Popular Culture, 27(1), 81-98. doi:10.1111/j.00223840.1993.845217931.x

Gentile, D., Anderson, C. A., Yukawa, S., Ihori, N., Saleem, M., Kam Ming, L., . . Sakamoto, A. (2009). The Effects of Prosocial Video Games on Prosocial Behaviors: International Evidence from Correlational, Longitudinal, and Experimental Studies. PubMed Central.

Getting Smart Research Center. (2017, June). How Minecraft Supports Social and Emotional Learning in K-12 Education. Retrieved from https://www.gettingsmart.com/wp-content/uploads/2017/04/How-MinecraftSupports-SEL.pdf

Glasscaseinpoint, . (2016, May 02). Playing the art market: Cultural capital in videogames. Retrieved April 19, 2021, from https://glasscaseinpoint.wordpress.com/2016/05/02/playing-the-art-marketcultural-capital-in-videogames/

Granic, I., Lobel, A., \& Engels, R. C. (n.d.). The Benefits of Playing Video Games. American Psychological Association,69(1), 66-78.

Greitemeyer, T., Traut-Mattausch, E., \& Osswald, S. (2012). How to ameliorate negative effects of violent video games on cooperation: Play it cooperatively in a team. Computers in Human Behavior, 28, 1465-1470. 
Grenfell, M. (2009). Applying Bourdieu's Field theory: The case of social capital and education. Education, Knowledge and Economy, 3(1), 17-34. doi:10.1080/17496890902786812

Halbrook, Y. J., O'Donnell, A. T., \& Msetfi, R. M. (2019). When and How Video Games Can Be Good: A Review of the Positive Effects of Video Games on Well Being. Association for Psychological Science, 14(6), 1096-1101.

Harker, R., Mahar, C., \& Wilkes, C. (1990). Chapter 1: Basic Theoretical Position. In An Introduction to the Work of Pierre Bourdieu (pp. 1-22). London, England: MacMillan Press.

Hopf, T. (1998). The Promise of Constructivism in International Relations Theory. International Security, 23(1), 171-200.

How to Trade in Rocket League? - Complete Guide. (n.d.). Retrieved from https://rl.exchange/blog/how-to-trade-in-rocket-league

Ihlen, Ø. (2018). Symbolic capital. The International Encyclopedia of Strategic Communication, 1-4. doi:10.1002/9781119010722.iesc0190

Jennifer Ouellette - Mar 16, 2. (2020, March 16). What a wow virtual outbreak taught us about how humans behave in epidemics. Retrieved March 02, 2021, from https://arstechnica.com/science/2020/03/that-time-world-of-warcraft-helpedepidemiologists-model-an-outbreak/

Korkeila, H., \& Hamari, J. (2020). Avatar capital: The relationships between player orientation and their avatar's social, symbolic, economic and cultural capital. Computers in Human Behavior, 102, 14-21. doi:10.1016/j.chb.2019.07.036 
Lau, K. J. (n.d.). The Political Lives of Avatars: Play and Democracy in Virtual Worlds. Western States Folklore Society, 69, 369-394.

Lee, Y. (2019). Older adults' digital gameplay, social capital, social connectedness, and civic participation. International Journal of Computer Game Research, 19(1).

Lefebvre, E., By, -, \& Lefebvre, E. (2019, September 13). Perfect Ten: The many modes of social experience in MMOs. Retrieved April 06, 2021, from https://massivelyop.com/2019/09/11/perfect-ten-the-many-modes-of-socialexperience-in-mmos/

Lenhart, A., Kahne, J., Middaugh, E., Rankin Macgill, A., Evans, C., \& Vitak, J. (2008). Teens, Video Games, and Civics: Teens' gaming experiences are diverse and include significant social interaction and civic engagement. Pew Internet \& American Life Project, 1-47.

Lopez, M. H., \& Moore, K. (2006). Participation in Sports and Civic Engagement. The Center for Information and Research on Civic Learning and Engagement, 1-9

Lowry, B. (2019, July 18). Everything you need to know about Overwatch's Role Queue system. Retrieved from https://www.windowscentral.com/everything-you-needknow-about-overwatch-role-queue-system

Maher, B. (2016, March 31). Can a Video Game Company Tame Toxic Behavior? Retrieved from https://www.nature.com/news/can-a-video-game-company-tametoxic-behaviour-1.19647 
Maher, C. (2020, March 18). This Minecraft library is making Censored journalism accessible all over the world. Retrieved April 19, 2021, from https://www.theverge.com/2020/3/18/21184041/minecraft-library-censoredjournalism-reporters-without-borders

Marone, V. (2016). Playful Constructivism: Making Sense of Digital Games for Learning and Creativity Through Play, Design, and Participation. Journal of Virtual Worlds Research, 9(3), 1-13.

Mäyrä, F. (2010). Gaming Culture at the Boundaries of Play. International Journal of Computer Game Research, 10(1).

Märtens, M., Shen, S., Iosup, A., \& Kuipers, F. (n.d.). Toxicity Detection in Multiplayer Online Games. 1-6.

McGonigal, J. (2011). Chapter 3: More Satisfying Work. In Reality is Broken: Why Games Make Us Better and How They Can Change the World (pp. 52-63). London: Penguin Press.

McQuarters, G. M. (2013). The Social Relations and Interactions of a First Person Shooter (FPS) Gamer (Master's thesis, University of Waikato, 2013) (pp. 36-56). Waikato: University of Waikato.

Michailidis, L., Balaguer-Ballester, E., \& He, X. (2018). Flow and immersion in video games: The aftermath of a conceptual challenge. Frontiers in Psychology, 9. doi:10.3389/fpsyg.2018.01682

Miller, T. (2019, June 24). 8 ways to make money playing video games. Retrieved April 19, 2021, from https://www.thestreet.com/personal-finance/how-to-make-moneyplaying-video-games-14996867 
Molyneux, L., Vasudevan, K., \& Gil de Zúñiga, H. (2015). Gaming social capital: Exploring civic value in multiplayer video games. Journal of Computer-Mediated Communication, 20(4), 381-399. doi:10.1111/jcc4.12123

Moncav, M. (2020, March 06). What is Elo? An explanation for competitive Gaming's Hidden rating system. Retrieved March 02, 2021, from https://dotesports.com/general/news/elo-ratings-explained-20565

Mosley, I. D. (2010). Personality and Character Selection in World of Warcraft (Master's thesis, Boise State University, 2010). Boise: Boise State University

Miura, S. (2007). Hierarchy: Social Science. Retrieved from https://www.britannica.com/topic/Great-Pacific-Garbage-Patch

Overwatch Staff. (n.d.). Competitive Play. Retrieved from https://overwatcharchive.fandom.com/wiki/Competitive_Play

Overwatch Staff. (n.d.). Endorsements. Retrieved from https://overwatch.fandom.com/wiki/Endorsements

Poor, N., \& Skoric, M. (2016). Play Together, Stay Together? Community Cohesion and Stability in an MMO. 49th Hawaii International Conference on System Sciences (pp. 2266-2274).

Rapp, A. (2018). Social game elements in world of Warcraft: Interpersonal Relations, groups, and organizations for Gamification design. International Journal of Human-Computer Interaction, 34(8), 759-773. 
Reddit User u/ttk2. (2013). You wake up in an unfamiliar place: A guide to Civcraft [Web $\log$ post]. Retrieved from https://www.reddit.com/r/Civcraft/comments/rmbgm/you_wake_up_in_an_unfam iliar_place_a_guide_to/ doi:10.1080/10447318.2018.1461760

Reer, F., \& Krämer, N. C. (2014). Underlying factors of social capital acquisition in the context OF online-gaming: Comparing world of Warcraft and Counter-Strike. Computers in Human Behavior, 36, 179-189. doi:10.1016/j.chb.2014.03.057

Rexhepi, A., Filiposka, S., \& Trajkovik, V. (2016). PLAYFUL E-PARTICIPATION WITH MINECRAFT AS DEVELOPMENT TOOL FOR URBAN REDESIGN: A CASE STUDY. International Conferences ICT, WBC, BIGDACI, and TPMC, 49-55.

Rigby, S., \& Ryan, R. (2011). The Emotional Experience of Games Today. In Glued to Games: How Video Games Draw Us in and Hold Us Spellbound (pp. 1-10). Westport, CT: Greenwood Publishing Group.

Risse, T. (2004). Social Constructivism Meets Globalization. Cambridge: Polity Press, 1-21. Ritz, I. E. (2006). Playing for an Active Community: Sports Participation and Civic Engagement (Master's thesis, Macalester College, 2006) (pp. 1-30). St. Paul: Digital Commons at Macalester College.

R. N. (2013). Chapter 2: Bourdieu's Forms of Capital and Video Game Production (1321376469 970383862 M. A. Ouellette, Ed.). In 1321376468970383862 J. C. Thompson (Ed.), The Game Culture Reader (pp. 30-46). Newcastle, UK: Cambridge Scholars Publishing. Rolfes, L., \& Passig, K. (2019). The Proto-Governance of Minecraft Servers. Journal of Virtual Worlds Research, 12(3), 1-12. 
Rossi, M. (2020, February 07). The social aspect of wow. Retrieved March 02, 2021, from https://www.engadget.com/2012-09-14-the-social-aspect-of-wow.html

Rotou, O., Qian, X., \& Von Davier, M. (2015, July). Ranking Systems Used in Gaming Assessments and/or Competitive Games [Scholarly project]. In Educational Testing Service Research Memorandum Series.

Ruggles, C., Wadley, G., \& Gibbs, M. R. (2005). Online community building techniques used by video game developers. Entertainment Computing - ICEC 2005, 114-125. doi:10.1007/11558651_12

Sanjamsai, S., \& Phukao, D. (2018). Flow experience in computer game playing among THAI university students. Kasetsart Journal of Social Sciences, 39(2), 175-182. doi: $10.1016 / j . k j s s .2018 .03 .003$

Seo, Y., \& Jung, S. (2014). Beyond solitary play in computer games: The social practices of eSports. Journal of Consumer Culture, 16(3), 635-655.

Seok, S., \& DaCosta, B. (2012). The world's most intense online gaming culture: Addiction and high-engagement prevalence rates among South Korean adolescents and young adults. Computers in Human Behavior, 28, 2143-2151.

Shaw, A. (2010). What is video Game Culture? Cultural studies and game studies. Games and Culture, 5(4), 403-424. doi:10.1177/1555412009360414

Shliakhovchuk, E., \& Muñoz Garcia, A. (2020). Intercultural perspective on impact of video games on players: Insights from a systematic review of recent literature. Educational Sciences: Theory \& Practice, 20(1), 40-58. doi:10.12738/jestp.2020.1.004 
Sims, C. (2014). Video game CULTURE, Contentious masculinities, and Reproducing Racialized social class divisions in middle school. Signs: Journal of Women in Culture and Society, 39(4), 848-857. doi:10.1086/675539

Social Statuses [Web log post]. (n.d.). Retrieved from https://tecfa.unige.ch/tecfa/maltt/VIP/Ressources/Articles/Game\%20Design\%20Patterns/ collection/Alphabetical_Patterns/SocialStatuses.htm

Soderberg-Rivkin, D. (2020, March 19). How Riot Games Used Behavior Science to Curb League of Legends Toxicity. Retrieved from https://www.spectrumlabsai.com/theblog/how-riot-games-is-used-behavior-science-to-curb-league-of-legends-toxicity

Stennson, E. (2009). The Social Structure of Massive Multiplayer Online Game Communities (Master's thesis, Stockholm University, 2009) (pp. 5-12). Stockholm: Stockholm University.

Toelstede, B. (2020). Social hierarchies in democracies and authoritarianism: The balance between power asymmetries and principal-agent chains. Rationality and Society, 32(3), 334-366. doi:10.1177/1043463120904051

Toft-Nielsen, C., \& Krogager, S. G. (2015). Gaming practices in everyday life. an analytical operationalization of field theory by means of practice theory. MedieKultur: Journal of Media and Communication Research, 31(58), 68. doi:10.7146/mediekultur.v31i58.18741

Von Heland, F., Westerberg, P., \& Nyberg, M. (2015). Future of Places: Emerging tools and tool kits. Manuscript, Stockholm, Sweden.

Williams, D., Ducheneaut, N., Xiong, L., Zhang, Y., Yee, N., \& Nickell, E. (2006). From tree house to barracks. Games and Culture, 1(4), 338-361. doi:10.1177/1555412006292616 
Williams, D. (2006). Groups and Goblins: The Social and Civic Impact of an Online Game. Journal of Broadcasting and Electronic Media, 50(4), 651-670.

Yee, N. (2006). The Labor of Fun: How Video Games Blur the Boundaries of Work and Play. Department of Communication, Stanford University, 1, 68-71.

Zendle, D., Ballou, N., \& Meyer, R. (2019). The changing face of desktop video game Monetization: An exploration of trends in loot boxes, pay to win, and cosmetic microtransactions in the Most-played Steam games Of 2010-2019. doi:10.31234/osf.io/u35kt

Zhang, F., \& Kaufman, D. (2015). Older adults' social interactions in massively multiplayer online role-playing games (mmorpgs). Games and Culture, 11(1-2), 150-169. doi:10.1177/1555412015601757

Zolyomi, A., \& Schmalz, M. (2017). Mining for Social Skills: Minecraft in Home and Therapy for Neurodiverse Youth. 50th Hawaii International Conference on System Sciences (pp. 3391-3399). 